\title{
Dopaminergic challenge dissociates learning from primary versus secondary sources of information
}

Rybicki, A. J.1, Sowden, S. L. 1 , Schuster, B. A. 1 \& Cook, J. $L_{1}$.

1. School of Psychology, University of Birmingham, Birmingham, B15 2TT, UK.

Correspondence concerning this article should be addressed to Alicia Rybicki, School of Psychology, University of Birmingham, Birmingham, B15 2TT, UK.

Phone: +44 7751865621; Email: axr783@bham.ac.uk 


\section{Summary}

2 Some theories of human cultural evolution posit that humans have social-specific learning

3 mechanisms that are adaptive specialisations moulded by natural selection to cope with the pressures of group living. However, the existence of neurochemical pathways that are specialised for learning from social information and from individual experience is widely debated. Cognitive neuroscientific studies present mixed evidence for social-specific learning mechanisms: some studies find dissociable neural correlates for social and individual learning whereas others find the same brain areas and, dopamine-mediated, computations involved in both. Here we demonstrate that,

9 like individual learning, social learning is modulated by the dopamine D2 receptor antagonist

10 haloperidol when social information is the primary learning source, but not when it comprises a

11 secondary, additional element. Two groups (total $\mathrm{N}=43$ ) completed a decision-making task which

12 required primary learning, from own experience, and secondary learning from an additional source.

13 For one group the primary source was social, and secondary was individual; for the other group this

14 was reversed. Haloperidol affected primary learning irrespective of social/individual nature, with no

15 effect on learning from the secondary source. Thus, we illustrate that neurochemical mechanisms 16 underpinning learning can be dissociated along a primary-secondary but not a social-individual axis.

17 These results resolve conflict in the literature and support an expanding field showing that, rather 18 than being specialised for particular inputs, neurochemical pathways in the human brain can process 19 both social and non-social cues and arbitrate between the two depending upon which cue is primarily relevant for the task at hand.

\section{Keywords}




\section{Introduction}

The complexity and sophistication of human learning is increasingly appreciated. Enduring theoretical models illustrate that learners utilise "prediction errors" to refine their predictions of future states (e.g. Rescorla-Wagner and temporal difference models; O'Doherty et al., 2003; Rescorla \& Wagner, 1972; Schultz et al., 1997; Sutton \& Barto, 2018). An explosion of studies, however, illustrates that this simple mechanism lies at the heart of more complex and sophisticated systems that enable humans (and other species) to learn from, keep track of the utility of, and integrate information from, multiple learning sources (Behrens et al., 2009; Biele et al., 2009; Li et al., 2011) meaning that one can learn from many sources of information simultaneously (Daw et al., 2006). Such complexity enables individuals to, for example, rank colleagues according to the utility of their advice and learn primarily from the top-ranked individual (Kendal et al., 2018; Laland, 2004; Morgan et al., 2012; Rendell et al., 2011) whilst also tracking the evolving utility of advice from others (Behrens et al., 2008; Biele et al., 2011). Recent studies have further revealed that learning need not rely solely on directly experienced associations, since one can also learn via inference (Bromberg-Martin et al., 2010; Dolan \& Dayan, 2013; Jones et al., 2012; Langdon et al., 2018; Moran et al., 2021; Sadacca et al., 2016; Sharpe \& Schoenbaum, 2018). This growing appreciation of the complexity and sophistication of human learning may help to explain contradictory findings in various fields. Here we focus on the field of social learning.

The existence in the human brain of neural and/or neurochemical pathways that are specialised for learning from social information and from individual experience respectively is the topic of much debate (Heyes, 2012; Heyes \& Pearce, 2015). Indeed, the claim that humans have social-specific learning mechanisms that are adaptive specialisations moulded by natural selection to cope with the pressures of group living, lies at the heart of some theories of cultural evolution (Kendal et al., 2018; Morgan et al., 2012; Templeton et al., 1999). Since cultural evolution is argued to be specific to humans (Richerson \& Boyd, 2005), establishing whether humans do indeed possess social-specific learning mechanisms has attracted many scholars with its promise of elucidating the key ingredient that "makes us human".

Cognitive neuroscience offers tools that are ideally suited to investigating whether the mechanisms underpinning social learning (learning from others), do indeed differ from those that govern learning from one's individual experience (individual learning). Cognitive neuroscientific studies, however, present mixed evidence for social-specific learning mechanisms. Some studies find dissociable neural 
correlates for social and individual learning (Apps et al., 2016; Behrens et al., 2008; Hill et al., 2016; Zhang \& Gläscher, 2020). For example, a study by Behrens and colleagues (2008) reported that whilst individual learning was associated with activity in dopamine-rich regions such as the striatum that are classically associated with reinforcement learning, social learning was associated with activity in a dissociable network that instead included the anterior cingulate cortex gyrus (ACCg) and temporoparietal junction. Further supporting this dissociation, studies have revealed correlations between personality traits, such as social dominance (Cook et al., 2014) and dimensions of psychopathy (Brazil et al., 2013) and social, but not individual, learning; as well as atypical social, but not individual, prediction error-related signals in the ACCg in autistic individuals (Balsters et al., 2017). Together these studies support the existence of social-specific learning mechanisms. In contrast, other studies have reported that the same computations, based on the calculation of prediction error, are involved in both social and individual learning (Diaconescu et al., 2014), and that social learning is associated with activity in dopamine-rich brain regions typically linked to individual learning (Biele et al., 2009; Braams et al., 2014; Campbell-Meiklejohn et al., 2010; Delgado et al., 2005; Diaconescu et al., 2017; Klucharev et al., 2009). Diaconescu and colleagues (2017), for example, observed that social learning-related prediction errors covaried with naturally occurring genetic variation that affected the function of the dopamine system. Further supporting this overlap between social and individual learning, behavioural studies have observed that social and individual learning are subject to the same contextual influences. For example, Tarantola and colleagues (2017) observed that prior preferences bias social learning, just as they do individual learning. Such findings promote the view that 'domain-general' learning mechanisms underpin social learning: we learn from other people in the same way that we learn from any other stimulus in our environment (Heyes, 2012; Heyes \& Pearce, 2015). That is, there are no social-specific learning mechanisms.

One potential resolution to this conflict in the literature hinges on i) an appreciation of the complexity and sophistication of human learning systems and ii) a difference in study design between tasks that have, and have not, found evidence of social-specific mechanisms. In studies, that have linked social learning with the dopamine-rich circuitry typically associated with individual learning (and which are therefore consistent with the domain general view), participants have been encouraged to learn primarily from social information. Indeed, in many cases the social source has been the sole information source (Campbell-Meiklejohn et al., 2017; Diaconescu et al., 2017;

89 Klucharev et al., 2009). For example, in the paradigm employed by Diaconescu and colleagues (2014, 90 2017), participants were required to choose between a blue and green stimulus and were provided with social advice which was sometimes valid and sometimes misleading; on each trial, participants 
received information about the time-varying probability of reward associated with the blue and green stimuli, thus participants did not have to rely on their own individual experience of blue/green reward associations and could fully dedicate themselves to social learning. That is, participants did not learn from multiple sources (i.e., social information and individual experience); participants only engaged in social learning. In contrast, in studies where social learning has been associated with neural correlates outside of the dopamine-rich regions classically linked to individual learning (and which are therefore consistent with the domain specific view), social information has typically comprised a secondary, additional source (Behrens et al., 2008; Cook et al., 2014). Typically, the nonsocial (individual) information is presented first to participants, represented in a highly salient form, and is directly related to the feedback information. The social information, in contrast, is presented second, is typically less salient in form, and is not directly related to the feedback information. For example, in the Behrens et al. study (2008) (and in our own work employing this paradigm (Cook et al., 2014, 2019)) participants were required to choose between two, highly salient, blue and green boxes to accumulate points. The boxes were the first stimuli that participants saw on each trial. Outcome information came in the form of a blue or green indicator thus primarily informing participants about whether they had made the correct choice on the current trial (i.e., if the outcome indicator was blue, then the blue box was correct). In addition, each trial also featured a thin red frame, which represented social information, surrounding one of the two boxes. The red frame was the second stimulus that participants saw on each trial and indirectly informed participants about the veracity of the frame: if the outcome was blue AND the frame surrounded the

112 blue box, then the frame was correct. In such paradigms, participants must learn from multiple

113 sources of information with one source taking primary status over the other. Consequently, in

114 studies that have successfully dissociated social and individual learning the two forms of learning

115 differ both in terms of social nature (social or non-social) and rank (primary versus secondary status).

116 Thus, it is unclear which of these two factors accounts for the dissociation.

118 The current study tests whether social and individual learning share common neurochemical

119 mechanisms when they are matched in terms of (primary versus secondary) status. Given its

120 acclaimed role in learning (Glimcher \& Bayer, 2005; Schultz, 2007), we focus specifically on the role

121 of the neuromodulator dopamine. Drawing upon recent studies illustrating the complexity and 122 sophistication of human learning (Daw et al., 2005; Gläscher et al., 2011; Moran et al., 2021) we 123 hypothesise that pharmacological modulation of the human dopamine system will dissociate 124 learning from two sources of information along a primary versus secondary, but not along a social 125 versus individual axis. In other words, we hypothesise that social learning relies upon the dopamine- 
rich mechanisms that also underpin individual learning when social information is the primary source, but not when it comprises a secondary, additional element. Such a finding would offer a potential resolution to the aforementioned debate concerning the existence of social-specific learning mechanisms.

Preliminary support for our hypothesis comes from three lines of work. First, studies have convincingly argued for flexibility within learning systems. For example, in a study by Daw and colleagues (2006), participants tracked the utility of four uncorrelated bandits, with particular brain

134 regions - such as the ventromedial prefrontal cortex - consistently representing the value of the top-

135 ranked bandit, even though the identity of this bandit changed over time. Second, studies are

136 increasingly illustrating the flexibility of social brain networks (Ereira et al., 2020; Garvert et al.,

137 2015). The medial prefrontal cortex (mPFC), for example, is not - as was once thought - specialised

138 for representing the self; if the concept of 'other' is primarily relevant for the task at hand, then the 139 mPFC will prioritise representation of other over self (Cook, 2014; Nicolle et al., 2012). Finally, in a 140 recent study (Cook et al., 2019), we provided preliminary evidence of a catecholaminergic (i.e. 141 dopaminergic and noradrenergic) dissociation between learning from primary and secondary, but 142 not social and individual, sources of information. In this work (Cook et al., 2019) we employed a 143 between-groups design, wherein both groups completed a version of the social learning task 144 adapted from Behrens and colleagues (2008; described above). For one group the secondary source 145 was social in nature (social group). For the non-social group, the secondary source comprised a

146 system of rigged roulette wheels and was thus non-social in nature. We observed that, in 147 comparison to placebo, the catecholaminergic transporter blocker methylphenidate only affected

148 learning from the primary source - which, in this paradigm, always comprised participant's own

149 individual experience. Methylphenidate did not affect learning from the secondary source,

150 irrespective of its social or non-social nature. That is, we found positive evidence supporting a

151 dissociation between primary and secondary learning but no evidence to support a distinction

152 between learning from social and non-social sources. Nevertheless, since we did not observe an

153 effect of methylphenidate on learning from the (social or non-social) secondary source of

154 information this study was unable to provide positive evidence of shared mechanisms for learning

155 from social and non-social sources. If it is truly the case that domain-general (neurochemical)

156 mechanisms underpin social learning, it should follow that pharmacological manipulations that

157 affect individual learning when individual information is the primary source also affect social learning 158 when social information is the primary source. 
The current (pre-registered) experiment tested this hypothesis by orthogonalizing social versus

160 individual and primary versus secondary learning. We perturbed learning using the dopamine D2 receptor antagonist haloperidol, in a double-blind, counter-balanced, placebo-controlled design. To test whether pharmacological manipulation of dopamine dissociates learning along a primarysecondary and/or a social-individual axis, we developed a novel between-groups manipulation wherein one group of participants learned primarily from social information and could supplement this learning with their own individual experience, and a second group learned primarily from individual experience and could supplement this learning with socially learned information. To foreshadow our results, we demonstrate that haloperidol specifically affects learning from the primary (not secondary) source of information. Bayesian statistics confirmed that the effects of haloperidol were comparable between the groups thus, haloperidol affected individual learning when individual information was the primary source and, to the same extent, social learning when social information was the primary source. Our data support an expanding field showing that, rather

172 than being fixedly specialised for particular inputs, neurochemical pathways in the human brain can

173 process both social and non-social cues and arbitrate between the two depending upon which cue is primarily relevant for the task at hand (Cook, 2014; Garvert et al., 2015; Nicolle et al., 2012).

Results females; see Methods) completed an adapted version of the behavioural task originally developed by Behrens and colleagues (Behrens et al., 2008). Participants were randomly allocated to one of two groups. Participants in the individual-primary group $(n=21)$ completed the classic version of this task (Figure 1A (Behrens et al., 2008)) in which they were required to make a choice between a

183 blue and green box in order to win points. A red frame (the social information), which represented

184 the most popular choice made by a group of four participants who had completed the task

185 previously, surrounded either the blue or green box on each trial and participants could use this to

186 help guide their choice. The actual probability of reward associated with the blue and green boxes

187 and the probability that the red frame surrounded the correct box varied according to uncorrelated

188 pseudo-randomised schedules (Figure S1; Appendix 2). For the individual-primary group, the

189 individual information (blue and green stimuli) was primary, and the social information (red

190 stimulus) was secondary on the basis that the blue/green stimuli appeared first on the screen, were

191 highly salient (large boxes versus a thin frame) and were directly related to the feedback

192 information. That is, after making their selection, participants saw a small blue or green box which 
193 primarily informed them whether a blue or green choice had been rewarded on the current trial.

194 From this information the participant could, secondarily, infer whether the social information (red

195 frame) was correct or incorrect.

197 Our social-primary group ( $n=22$; groups matched on age, gender, body mass index (BMI) and verbal

198 working memory span (Table 1)) completed an adapted version of this task (Figure 1B) wherein the

199 social information (red stimulus) was primary, and the individual information (blue/green stimuli)

200 was secondary. Participants first saw two placeholders; one empty and one containing a red box

201 which indicated the social information. Subsequently, a thin green and a thin blue frame appeared

202 around each placeholder. Participants were told that the red box represented the group's choice.

203 They were then required to choose whether to go with the social group (red box) or not. After

204 making their choice a tick or cross appeared which primarily informed participants whether going

205 with the social information was the correct option. From this they could, secondarily, infer whether

206 the blue or green frame was correct. Consequently, for the social-primary group the social

207 information was primary on the basis that it appeared first on the screen, was highly salient (a large

208 red box versus thin green/blue frames) and was directly related to the feedback information.

210 Participants in both the individual-primary and social-primary groups performed 120 trials of the

211 task on each of two separate study days. To perturb learning, on one day participants took $2.5 \mathrm{mg}$ of

212 haloperidol (HAL), previously shown to affect learning (Pessiglione et al., 2006) via multiple routes

213 including perturbation of phasic dopamine signalling (Schultz, 2007; Schultz et al., 1997) facilitated

214 by action at mesolimbic D2 receptors (Camps et al., 1989; Grace, 2002; Lidow et al., 1991). On the

215 other day, they took a placebo (PLA) under double-blind conditions, with the order of the days

216 counterbalanced. 43 participants took part in at least one study day, 33 participants completed both

217 study days. 2 participants performed at below chance level accuracy and were excluded from further

218 analysis. We present an analysis of data from the 31 participants who completed both study days

219 with above chance accuracy (Table 1 ) in the main text of this manuscript, which we complement

220 with a full analysis of all 41 datasets in Appendix $4 \mathrm{i}$. 
Figure 1. Behavioural task
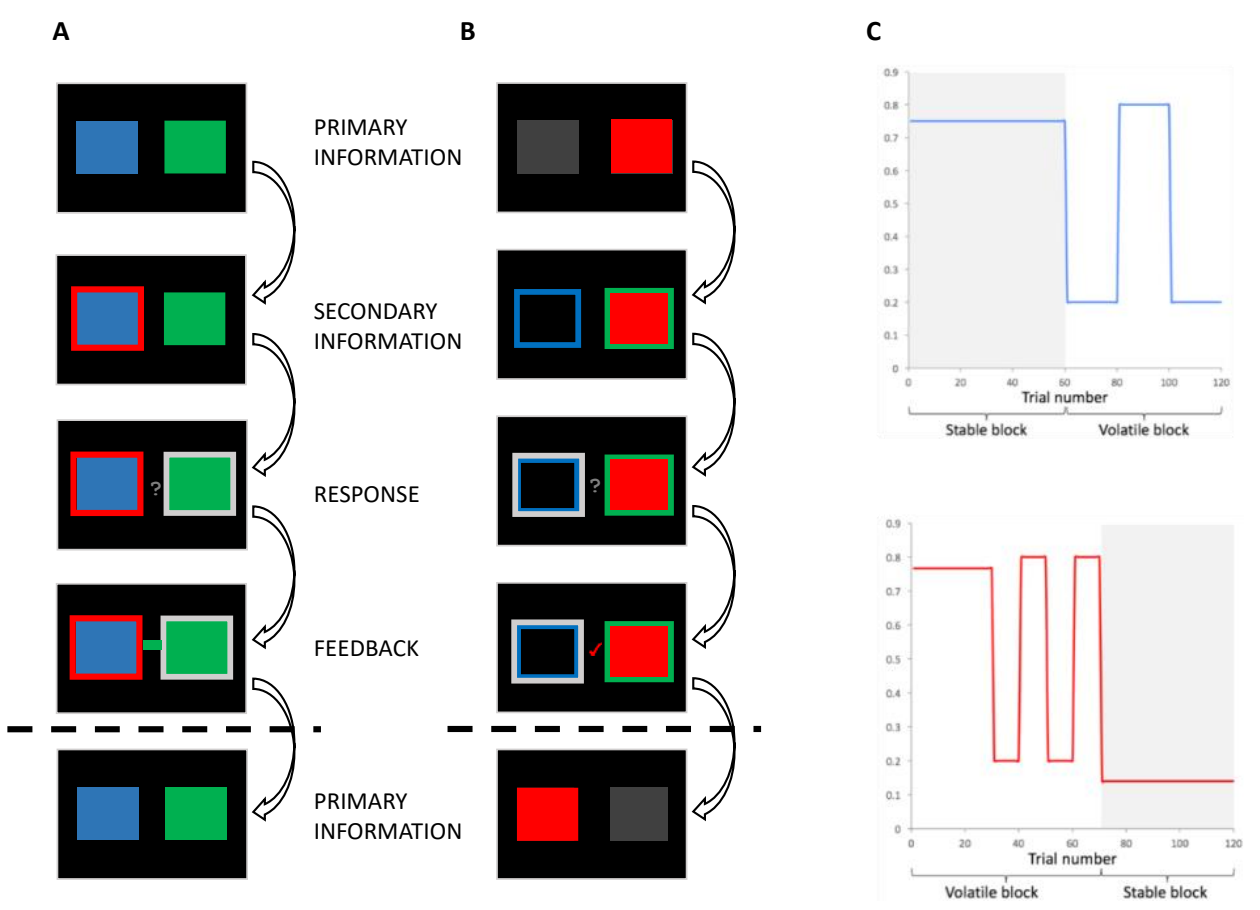

Figure 1. Behavioural task. A. Individual-primary group. Participants selected between a blue and a green box to gain points. On each trial, the blue and green boxes were presented first. After 1-4 seconds (s), one of the boxes was highlighted with a red frame, representing the social information. After $0.5-2 \mathrm{~s}$, a question mark appeared, indicating that participants were able to make their response. Response was indicated by a silver frame surrounding their choice. After a 1-3s interval, participants received feedback in the form of a green or blue box in the middle of the screen. B. Social-primary group. Participants selected between going with, or against a red box, which represented the social information. On each trial, the red box was displayed. After 1-4s, blue and green frames appeared. After 0.5-2s, a question mark appeared, indicating that participants were able to make their response. Response was indicated by a silver frame surrounding their choice. After a 1-3s interval, participants received feedback in the form of a tick or a cross. This feedback informed participants if going with the group was correct or incorrect, from this feedback participants could infer whether the blue or green frame was correct. C. Example of pseudo-randomised probabilistic schedule. The probability of reward varied according to probabilistic schedules, including stable and volatile blocks for both the probability of the blue box/frame being correct (top) and the probability of the red (social) box/frame being correct (bottom).

Social information is the primary source of learning for participants in the social-primary group

222 Our novel manipulation orthogonalized primary versus secondary and social versus individual

223 learning. To validate our manipulation, we tested whether participants in both the individual-

224 primary and social-primary group learned in a more optimal fashion from the primary versus secondary source of information in our placebo condition. For this validation analysis we used a Bayesian learner model to create two optimal models (1) an optimal primary learner, and (2) an 
choice data, resulting in two $\beta_{\text {optimal }}$ values capturing the extent to which a participant made choices according to the optimal primary, and optimal secondary learner models respectively. $\beta_{\text {optimal }}$ values were submitted to a repeated-measures ANOVA with factors information source (primary, secondary) and group (social-primary, individual-primary), revealing main effects of information source and group. $\beta_{\text {optimal }}$ values were significantly higher for the primary information $\left(\bar{x}\left(\sigma_{\bar{x}}\right)=0.872\right.$

$233(0.101))$, compared with secondary information source $\left(\bar{x}\left(\sigma_{\bar{x}}\right)=0.438(0.101) ; \mathrm{t}(30)=2.568\right.$, pholm $=$

234 0.016). $\beta_{\text {optimal }}$ values were also significantly higher for the social-primary $\left(\bar{x}\left(\sigma_{\bar{x}}\right)=0.833(0.078)\right.$ ),

235 compared with the individual-primary group $\left(\bar{x}\left(\sigma_{\bar{x}}\right)=0.477(0.078) ; \mathrm{t}(30)=3.228\right.$, pholm $\left.=0.003\right)$

236 (Figure 2). Crucially, we did not observe a significant interaction between information and group ( $F$ $237(1,29)=0.067, p=0.797)$, meaning that participants' choices were more influenced by the primary 238 information source, regardless of whether it was social or individual in nature. Furthermore, $\beta_{\text {optimal }}$ 239 values for primary information did not differ between groups $(t(29)=-1.211, p=0.236)$. Note that, $240 \beta_{\text {optimal }}$ weights for both information sources were significantly greater than zero (primary: $\mathrm{t}(30)=$ $2415.534, p<0.001$; secondary: $\mathrm{t}(30)=4.789, \mathrm{p}<0.001$ ) thus our optimal models of information use

242 explained a significant amount of variance in the use of both primary and secondary learning

243 sources. These data show that, irrespective of social (or individual) nature, participants learned in a 244 more optimal fashion from the "primary" (relative to secondary) learning source, which was first in 245 the temporal order of events, highly salient and directly related to the reward feedback. 
Figure 2. Beta weights ( $\beta$ _optimal)

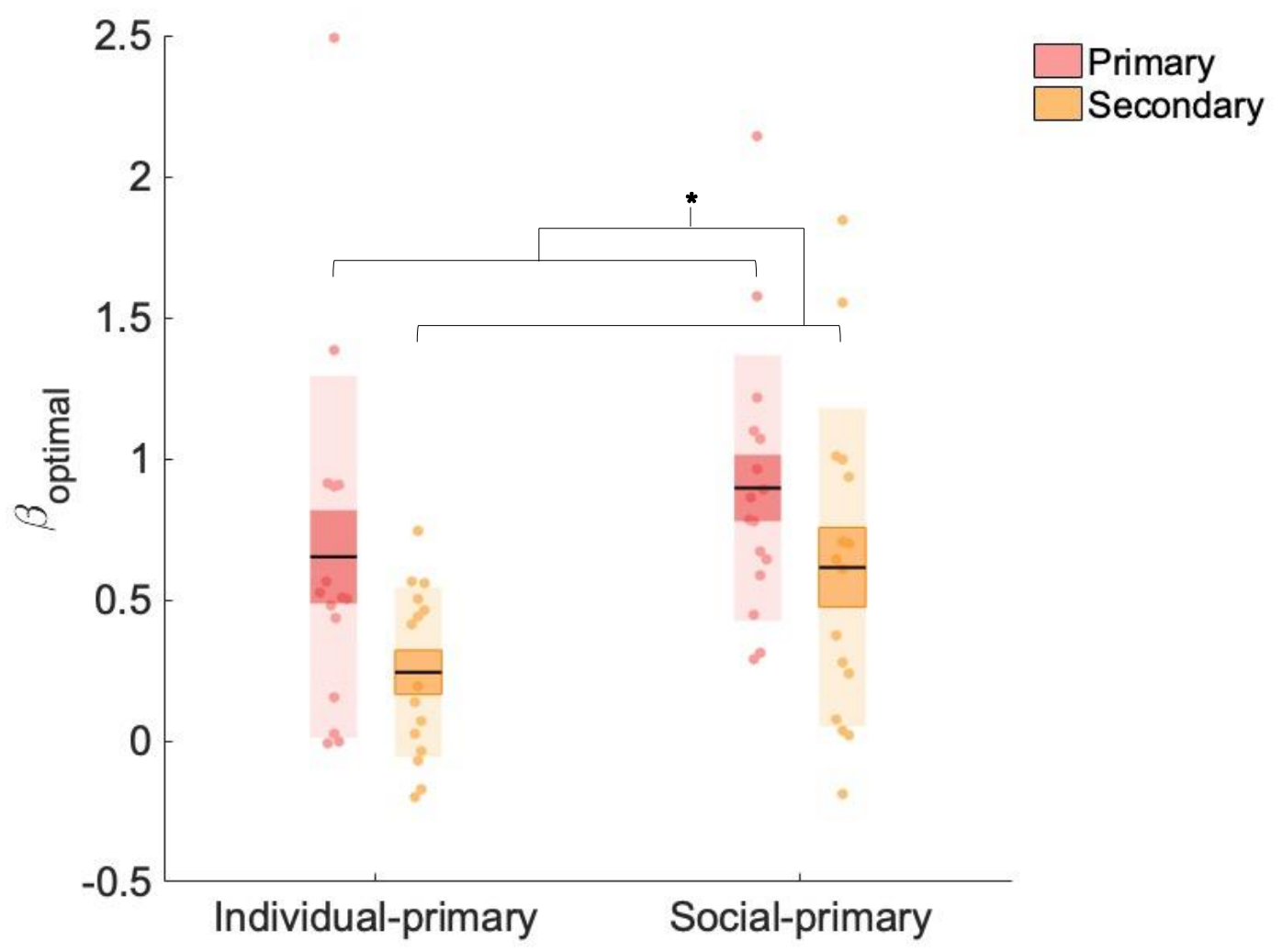

Figure 2. Beta weights ( $\beta$ _optimal) for primary and secondary information. Data points indicate estimated $\beta$ _optimal weights for individual participants $(n=31$, placebo data), bold point indicates the mean, bold line indicates standard error of the mean (1 SEM), * indicates statistical significance $(p<0.05)$.

\section{Haloperidol reduces the rate of learning from primary sources}

247 We hypothesed that both social and individual learning would be modulated by administration of

248 the dopamine D2 receptor antagonist haloperidol when they were the primary source of learning,

249 but not when they comprised the secondary source. To test this hypothesis we fitted an adapted

250 Rescorla-Wagner (RW) learning model (Rescorla \& Wagner, 1972) to participants' choice data, enabling us to estimate various parameters that index learning from primary and secondary sources of information, for HAL and PLA conditions, for participants in the social-primary and individualprimary groups. Our adapted RW model provided estimates, for each participant, of $\alpha, \beta$, and $\zeta$. The learning rate $(\alpha)$ controls the weighting of prediction errors on each trial. A high $\alpha$ favours recent over (outdated) historical outcomes, while a low $\alpha$ suggests a more equal weighting of recent and more distant trials. Since our pseudo-random schedules included stable phases (where the reward probability associated with a particular option was constant for $>30$ trials), and volatile phases 
(where reward probabilities changed every 10-20 trials), $\alpha$ was estimated separately for volatile and stable phases (for both primary and secondary learning) to accord with previous research (Behrens et al., 2007; Cook et al., 2019; Manning et al., 2017). $\beta$ captures the extent to which learned probabilities determine choice, with a larger $\beta$ meaning that choices are more deterministic with regard to the learned probabilities. $\zeta$ represents the relative weighting of primary and secondary sources of information, with higher values indicating a bias towards the over-weighting of secondary relative to primary (see Methods and Appendix 3 for further details of the model, model fitting and model comparison).

To test the hypothesis that haloperidol would affect learning from the primary information source only, regardless of its social/individual nature, we employed three separate linear mixed effects models, allowing analysis of the effects of fixed factors information source (primary, secondary), drug (HAL, PLA), environmental volatility (volatile, stable) and group (social-primary, individualprimary) on our three dependent variables $(\alpha, \beta, \zeta)$ while controlling for inter-individual differences. Including pseudo-randomisation schedule as a factor in all analyses did not change the pattern of results. A repeated measures ANOVA (RM-ANOVA) on mixed effects model coefficients revealed no main/interaction effect(s) on $\beta$ or $\zeta$ values (all $p>0.05$ ). In contrast, for $\alpha$ we observed a drug by information interaction $\left(F(1,203)=6.852, p=0.009\right.$, beta estimate $\left(\sigma_{\bar{x}}\right)=0.026(0.010), t=2.62$, confidence interval $[\mathrm{CI}][0.010-0.050]$ ) (Figure 3). There were no significant main effects of drug ( $\mathrm{F}$ $(1,258)=0.084, p=0.772)$, group $(F(1,39)=3.692, p=0.062)$ or volatility $(F(1,258)=0.084, p=$ 0.772 ) on $\alpha$ values, nor any other significant interactions involving drug (all $p$-values $>0.05$, see Appendix $4 \mathrm{v}$-vi for analysis including schedule, session and working memory). Planned contrasts showed that, whilst under PLA $\alpha_{\text {primary }}\left(\bar{x}\left(\sigma_{\bar{x}}\right)=0.451(0.025)\right)$ was significantly greater than $\alpha_{\text {secondary }}$ $\left(\bar{x}\left(\sigma_{\bar{x}}\right)=0.370(0.025) ; z(30)=2.861, \mathrm{p}=0.004\right)$, this was not the case under HAL $\left(\alpha_{\text {primary }} \bar{x}\left(\sigma_{\bar{x}}\right)=\right.$ $\left.0.393(0.025), \alpha_{\text {secondary }} \bar{x}\left(\sigma_{\bar{x}}\right)=0.417(0.025) ; z(30)=-0.843, \mathrm{p}=0.400\right)$. Furthermore, $\alpha_{\text {primary }}$ was decreased under HAL relative to PLA ( $z(30)=-2.050, p=0.040)$. Although $\alpha_{\text {secondary }}$ was, in contrast, numerically increased under $\operatorname{HAL}\left(\bar{x}\left(\sigma_{\bar{x}}\right)=0.417(0.025)\right.$ relative to PLA $\left(\bar{x}\left(\sigma_{\bar{x}}\right)=0.370(0.025)\right.$, this difference was not significant $(z(30)=1.654, p=0.098)$. This drug $x$ information interaction therefore illustrated that whilst haloperidol significantly reduced $\alpha_{\text {primary }}$ it had no significant effect on $\alpha_{\text {secondary. }}$ Furthermore, under PLA there was a significant difference between $\alpha_{\text {primary }}$ and $\alpha_{\text {secondary, }}$ which was nullified by haloperidol administration. Consequently, under placebo participants' rate of learning was typically higher for learning from the primary relative to the secondary source, however, under the D2 receptor antagonist haloperidol the rate of learning from the primary source 
291 was reduced and thus there was no significant difference in the rate of learning from primary and 292 secondary sources.

Figure 3. Learning rate estimates

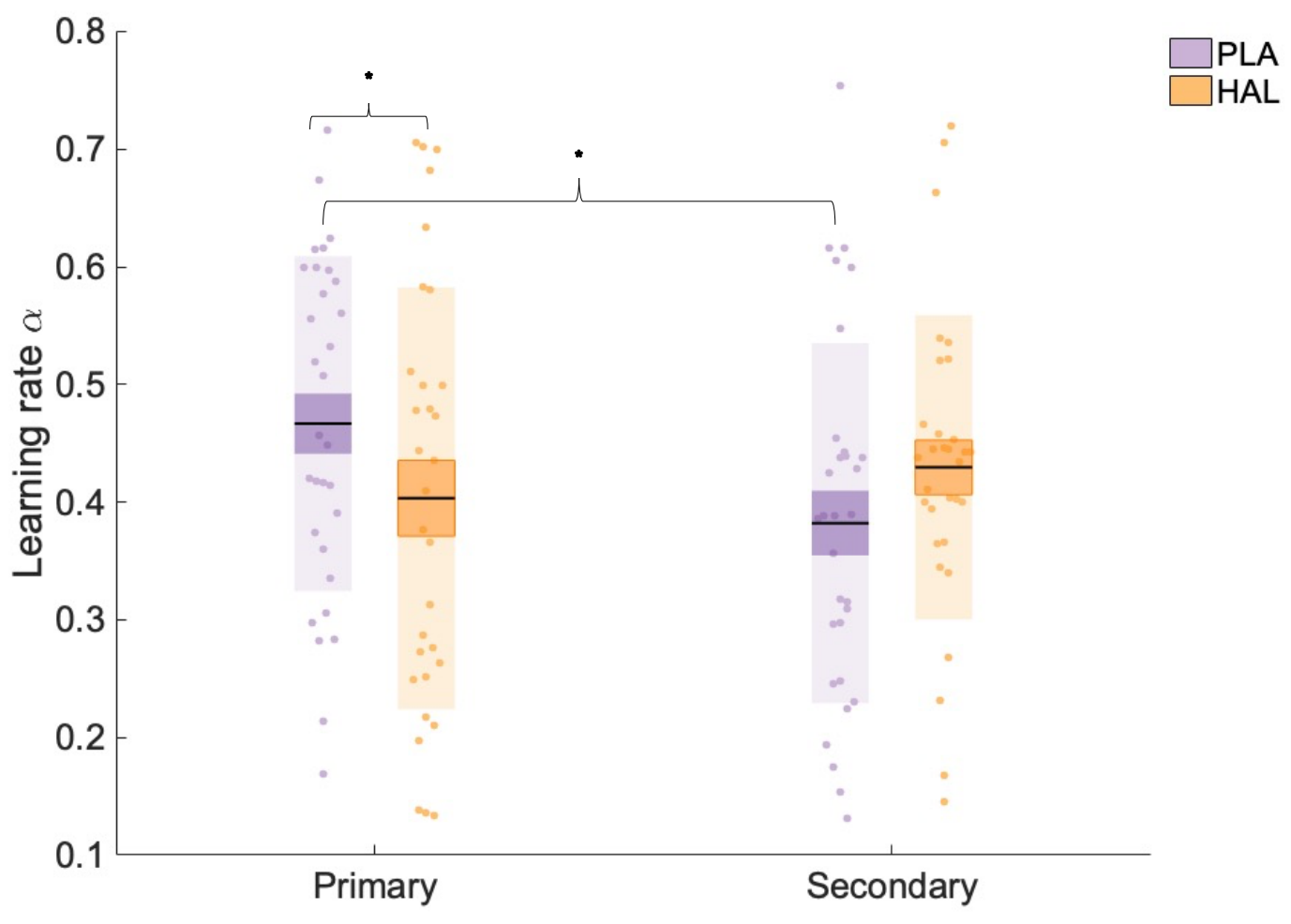

Figure 3. Learning rate $(\alpha)$ estimates for learning from primary and secondary information. There was a significant interaction between information and drug, with $\alpha$ estimates significantly lower under haloperidol (orange), relative to placebo (purple), for primary information only. Data points indicate square-root transformed $\alpha$ estimates for individual participants $(n=31)$, boxes $=$ standard error of the mean, shaded region $=$ standard deviation, $\mathrm{HAL}=$ haloperidol, PLA = placebo, $*$ indicates statistical significance $(p<0.05)$.

\section{Haloperidol reduces the rate of learning from a primary source irrespective of its social or}

\section{4 individual nature}

295 Our primary hypothesis was that haloperidol would modulate the rate of learning from the primary

296 source irrespective of its social or individual nature. This would be evidenced as an interaction

297 between drug and (primary versus secondary) information source (see above) in the absence of an

298 interaction between drug, information source and group (social-primary versus individual-primary).

299 Crucially, we observed no significant interaction between drug, information source and group (F (1,

$300234)=0.029, p=0.866$ ). To further assess whether drug effects on primary information differed as a

301 function of group, results were also analysed within a Bayesian framework, using JASP software 
302 (JASP Team (2020)). A Bayes exclusion factor ( $\left.\mathrm{BF}_{\text {excl}}\right)$, representing the relative likelihood that a

303 model without a drug $\mathrm{x}$ information $\mathrm{x}$ group interaction effect could best explain the observed data,

304 was calculated (Dienes, 2014). Values of 3-10 are taken as moderate evidence in favour of the null

305 hypotheses that there is no drug $\mathrm{x}$ information $\mathrm{x}$ group interaction (Lee \& Wagenmakers, 2013) with

306 values greater than 10 indicating strong evidence. The $\mathrm{BF}_{\text {excl }}$ value was equal to 7.516 , providing

307 moderate evidence in favour of the null hypotheses that there is no drug $\mathrm{x}$ information $\mathrm{x}$ group

308 interaction. Consequently, results confirmed our hypothesis: haloperidol perturbed learning from

309 the primary but not the secondary source, irrespective of social or individual nature.

310

\section{Haloperidol brings $\boldsymbol{\alpha}_{\text {primary }}$ estimates within the optimal range}

312 To assess whether the effects of haloperidol on $\alpha_{\text {primary }}$ are harmful or beneficial with respect to

313 performance we first explored drug effects on accuracy (see Appendix 4ii for a detailed analysis

314 including randomisation schedule). There was no significant difference in accuracy between

315 haloperidol $\left(\bar{x}\left(\sigma_{\bar{x}}\right)=0.600(0.013)\right)$, and placebo $\left(\bar{x}\left(\sigma_{\bar{x}}\right)=0.611(0.010) ; \mathrm{F}(1,29)=0.904, \mathrm{p}=0.349\right.$,

$\left.316 \eta_{\mathrm{p}}{ }^{2}=0.030\right)$ conditions.

318 The lack of a significant main effect of drug on accuracy was somewhat surprising given the

319 significant (interaction) effect on learning rates, i.e., a decrease in $\alpha_{\text {primary }}$ under haloperidol relative

320 to placebo. To investigate whether haloperidol resulted in learning rates that were less, or

321 alternatively more, optimal we compared our estimated $\alpha$ values with optimal $\alpha$ estimates. Since

322 trial-wise outcomes were identical to those utilised by Cook et al (Cook et al., 2019), optimal values

323 are also identical and are described here for completeness. An optimal learner model, with the same

324 architecture and priors as the model employed in the current task, was fit to 100 synthetic datasets,

325 resulting in average optimal learning rates: $\alpha_{\text {optimal_primary_stable }}=0.16, \alpha_{\text {optimal_primary_volatile }}=0.21$,

$326 \alpha_{\text {optimal_secondary_stable }}=0.17, \alpha_{\text {optimal_secondary_volatile }}=0.19$. Scores representing the difference between

327 (untransformed) $\alpha$ estimates and optimal $\alpha$ scores were calculated $\left(\alpha_{\text {diff }}=\alpha-\alpha_{\text {optimal }}\right)$. A linear

328 mixed model analysis on $\alpha_{\text {diff }}$ values with factors group, drug, volatility and information source and

329 subject as a random factor, was conducted. A RM-ANOVA (factors: drug, information, volatility,

330 group) on model coefficients revealed an interaction between drug and information source ( $F(1$,

$331203)=4.895, p=0.028$ ) (Figure 4). Separate RM-ANOVAs were conducted for primary and secondary

332 information. For primary information, a main effect of drug was observed on difference scores ( $F(1$,

$\left.33329)=51.740, \mathrm{p}<0.001, \mathrm{n}_{\mathrm{p}}{ }^{2}=0.641\right)$, with $\alpha_{\text {diff_primary }}$ significantly higher under PLA $\left(\bar{x}\left(\sigma_{\bar{x}}\right)=0.238\right.$

$334(0.026))$ compared with HAL $\left(\bar{x}\left(\sigma_{\bar{x}}\right)=0.011(0.026)\right)$. For secondary information, $\alpha_{\text {diff_secondary }}$ did

335 not differ between treatment conditions $(p>0.05)$. In sum, learning rates for learning from the 
336 primary source were higher than optimal under placebo, with $\alpha_{\text {diff_primary }}$ significantly differing

337 from 0 (one-sample $t$ test; $\mathrm{t}(30)=2.377, \mathrm{p}=0.024$ ). Haloperidol reduced learning rates that

338 corresponded to learning from the primary source, thus bringing them within the optimal range,

339 with $\alpha_{\text {diff_primary }}$ not significantly differing from 0 under haloperidol (one-sample $\mathrm{t}$ test; $\mathrm{t}(30)=$

$340 \quad 0.412, p=0.683)$. Consequently, under haloperidol relative to placebo, learning rates were more

341 optimal when learning from primary sources.

Figure 4. Learning rate estimates compared with optimal learning rates.

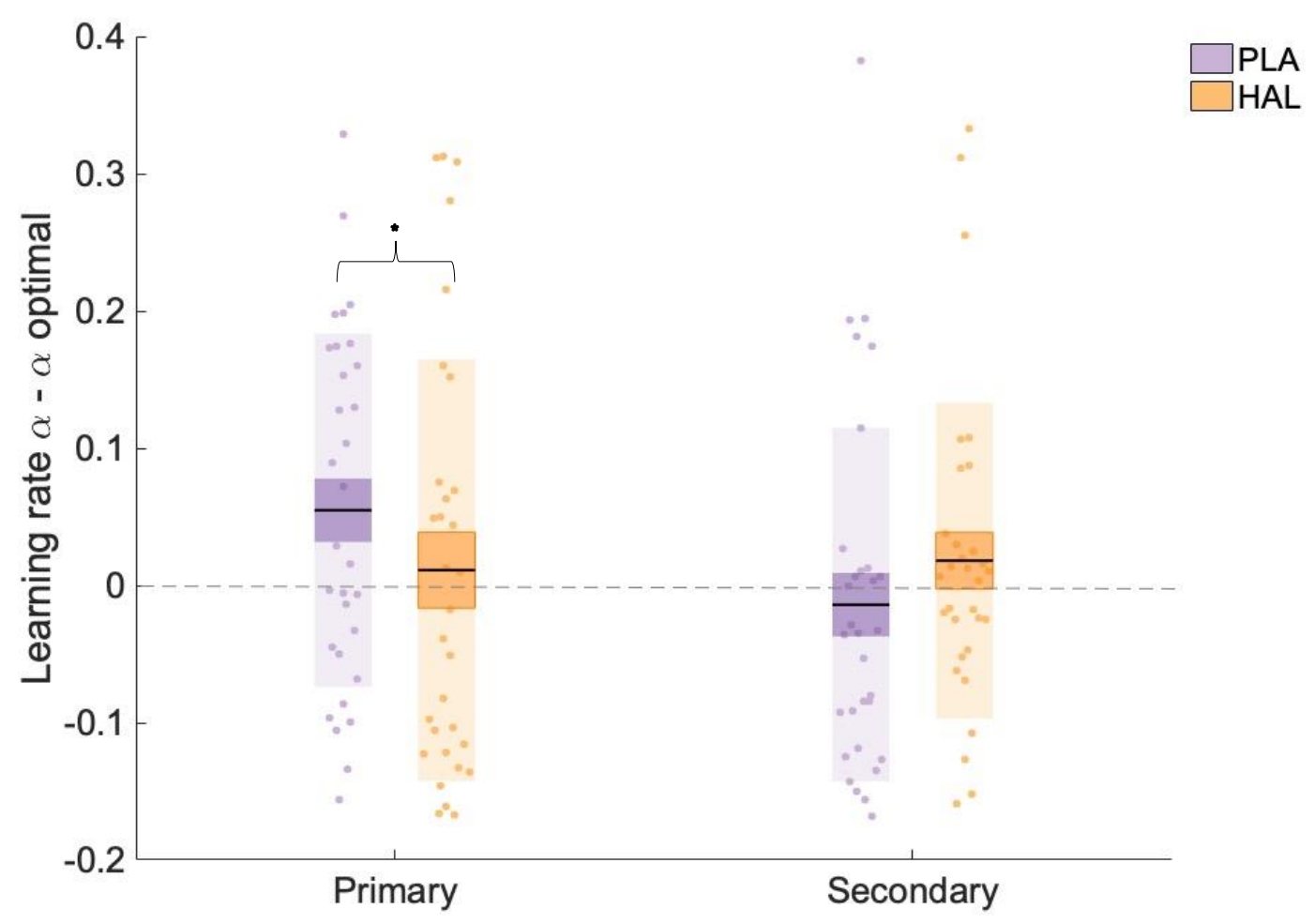

Figure 4. Learning rate estimates minus optimal learning rates. There was a significant interaction between information and drug, with $\alpha_{\text {primary }}$ scores significantly higher than optimal estimates under placebo but not under haloperidol. Data points indicate $\alpha-\alpha$ optimal values for individual participants (n $=31$ ), boxes = standard error of the mean, shaded region = standard deviation, $\mathrm{HAL}=$ haloperidol, PLA = placebo, * indicates statistical significance $(p<0.05)$.

342 To explore whether $\alpha$ values were in some way related to accuracy scores we used two separate

343 backwards regression models, for PLA and HAL conditions separately, with $\alpha_{\text {primary }}$ and $\alpha_{\text {secondary }}$ as

344 predictors and accuracy as the dependent variable (see Appendix 4iii for details of a regression

345 model with all model parameters). PLA accuracy was predicted by $\alpha_{\text {secondary }}$ though this model only

346 approached significance $(R=0.121, F(1,29)=3.981, p=0.055)$. Under HAL however, accuracy was 
347 predicted by a model with $\alpha_{\text {secondary }}$ and $\alpha_{\text {primary }}(R=0.450, F(2,28)=3.560, p=0.042)$, with $\alpha_{\text {primary }}$ a

348 significant positive predictor of accuracy $(\beta=0.404, p=0.028)$. Removing $\alpha_{\text {secondary }}$ as a predictor did

349 not significantly improve the fit of this model $\left(R^{2}\right.$ change $=0.014$, $F$ change $\left.(1,29)=0.495, p=1.000\right)$.

350 When combined with our optimality analysis, these results suggest that under placebo $\alpha_{\text {primary }}$ was

351 outside of the optimal range of $\alpha$ values and thus accuracy was primarily driven by $\alpha_{\text {secondary }}$.

352 However, haloperidol reduced $\alpha_{\text {primary, }}$ bringing it within the optimal range. Thus, under haloperidol

353 accuracy was driven by both $\alpha_{\text {primary }}$ and $\alpha_{\text {secondary. }}$

355 In sum, relative to placebo, the dopamine D2 receptor antagonist haloperidol significantly decreased

356 learning rates relating to learning from primary, but not secondary sources of information, likely via

357 mediation of phasic dopaminergic signalling (see Appendix 4iv). Interestingly, learning rates for

358 learning from the primary source were higher than optimal under placebo and haloperidol brought

359 them within the optimal range. Consequently, both primary and secondary learning contributed to

360 accuracy under haloperidol but not under placebo. Importantly, the effects of haloperidol did not

361 vary as a function of group allocation which dictated whether the primary source was of social or

362 individual nature. A Bayesian analysis confirmed that we had moderate evidence to support the 363 conclusion that there was no interaction between drug, learning source and group. These data, thus,

364 illustrate a dissociation along the primary-secondary but not social-individual axis.

Discussion

The current study tested the hypothesis that social and individual learning share common neurochemical mechanisms when they are matched in terms of (primary versus secondary) status. Specifically, we predicted that haloperidol would perturb learning from the primary but not the secondary source, irrespective of social or individual nature. Supporting our hypothesis, we observed an interaction between drug and information source (social versus individual) such that under haloperidol (compared to placebo) participants exhibited reduced learning rates with respect to learning from the primary, but not the secondary, source of information. Crucially, we did not observe an interaction between drug, information source and group (social-primary versus individual-primary). Bayesian statistics revealed that, given the observed data, a model that excludes this interaction is 7.5 times more likely than models which include the interaction. individual learning could be explained by participants not fully appreciating the social nature of the 
red shape (the social information source). In opposition to this, we argue that since our participants could not commence the task until reaching $100 \%$ accuracy in a pre-task quiz, which questioned participants about the social nature of the red shape, we can be confident that all participants knew that the red shape indicated information from previous participants. Participants also completed a post-task questionnaire (Appendix 5), which required them to reflect upon the extent to which their decisions were influenced by the social (red shape) and individual (blue/green shapes) information. The individual-primary and social-primary groups did not differ in their beliefs about the extent to which they were influenced by these two sources of information. Furthermore, in our previous work, using the same social manipulation, we demonstrated that the personality trait social dominance significantly predicts social, but not individual, learning (Cook et al., 2014). Thus, illustrating that participants treat the social information differently from the non-social information in this type of paradigm. Finally, based on previous studies, we argue that even with a more overtly social manipulation it is highly likely that social learning would still be perturbed by dopaminergic modulation when social information is the primary source. Indeed, in a study by Diaconescu et al.(2017) social information was represented by a video of a person indicating one of the two options. Even with this overtly social stimulus, Diaconescu et al. still observed that social learning covaried with genetic polymorphisms that affect the functioning of the dopamine system.

Our results comprise an important contribution to the debate concerning the existence of socialspecific learning mechanisms. We find that, like individual learning, social learning is modulated by a dopaminergic manipulation when it is the primary source of information. This result marries well with previous studies that have linked social learning with dopamine-rich mechanisms when the social source has been the primary (or in many cases the sole) information source (CampbellMeiklejohn et al., 2017; Diaconescu et al., 2017; Klucharev et al., 2009). Our results are also consistent with studies that have associated social learning with different neural correlates, outside of the dopamine-rich regions classically linked to individual learning, when it is a secondary source of information (Behrens et al., 2008; Hill et al., 2016; Zhang \& Gläscher, 2020). Our data suggest that social and individual learning share common dopaminergic mechanisms when they are the primary learning source and that previous dissociations between these two learning types may be more appropriately thought of as dissociations between learning from a primary and secondary source.

411 Extant studies (e.g. Cook et al., 2019) were not able to illustrate the importance of the primary 412 versus secondary distinction because they did not fully orthogonalize primary versus secondary and 413 social versus individual learning. 
415 Though our results suggest shared neurochemical mechanisms for social and individual learning

416 when they are matched in status, it is, nevertheless, essential to highlight that it does not follow that

417 there are no dimensions along which social learning may be dissociated from individual learning. For

418 instance, it is possible that although social and individual learning are affected by dopaminergic

419 modulation - when they are the primary source - there are differences in the location of neural

420 activity that could be revealed by neuroimaging. For instance, although social and individual learning

421 are both associated with activity within the striatum (Burke et al., 2010; Cooper et al., 2012), social-

422 specific activation patterns have been observed in other brain regions, including the temporoparietal

423 junction (Behrens et al., 2008; Lindström et al., 2018) and the gyrus of the anterior cingulate cortex

424 (Behrens et al., 2008; Hill et al., 2016; Zhang \& Gläscher, 2020). Such a location-based dissociation

425 requires further empirical investigation as well as further consideration of the possible functional

426 significance of such location-based differences, if they are indeed present when primary versus

427 secondary status is accounted for. Additionally, since we did not observe significant effects of

428 haloperidol on learning from social or individual sources when they were secondary in status, it

429 remains a logical possibility that social and individual learning can be neurochemically dissociated

430 when they are the secondary source of information - though it is admittedly difficult to conceive of a

431 parsimonious explanation for the existence of two neurochemical mechanisms for social and

432 individual learning from secondary sources. Finally, it is possible that social and individual learning

433 share common dopaminergic mechanisms when they are the primary source, but differentially

434 recruit other neurochemical systems. For instance, some have argued that social learning may

435 heavily rely upon serotonergic mechanisms (Crişan et al., 2009; Frey \& McCabe, 2020; Roberts et al.,

436 2020). The abovementioned avenues should be further explored however, in the interim, it must be

437 concluded that since existing studies have not controlled for primary versus secondary status, we do

438 not currently have convincing evidence that social and individual learning can be dissociated in the

439 human brain.

440

441 Notably, our results reveal a clear dissociation between learning from primary and secondary

442 sources. The effects of haloperidol on learning from the primary source are consistent with previous

443 work. Non-human animal studies, have shown that phasic signalling of dopaminergic neurons in the

444 mesolimbic pathway encodes reward prediction error signals (Schultz, 2007; Schultz et al., 1997).

445 Since haloperidol has high affinity for D2 receptors (Grace, 2002), which are densely distributed in

446 the mesolimbic pathway (Camps et al., 1989; Lidow et al., 1991), dopamine antagonists including

447 haloperidol can affect phasic dopamine signals (Frank and O'Reilly, 2006) - either via binding at

448 postsynaptic D2 receptors (which blocks the effects of phasic dopamine bursts), or via pre-synaptic 
449 autoreceptors (which has downstream effects on the release and reuptake of dopamine and thus 450 modulates bursting itself) (Benoit-Marand et al., 2001; Ford, 2014; Schmitz et al., 2003). Indeed a 451 number of studies have shown that haloperidol can attenuate prediction error-related signals

452 (Diederen et al., 2017; Haarsma et al., 2018; Menon et al., 2007; Pessiglione et al., 2006). In line with

453 this, we observed that learning rates were lower under haloperidol. However, in our paradigm

454 learning rates for learning from the primary source were higher than optimal under placebo, thus

455 haloperidol had the beneficial effect of bringing learning rates closer to optimal. In sum, our results

456 are in accordance with previous work demonstrating the importance of phasic dopamine D2-related

457 signalling in learning from primary sources.

459 Perhaps the most novel contribution of our work is that we here illustrate that, whilst dopaminergic

460 modulation affects learning from the primary source, it does not significantly affect learning from

461 the secondary source. Previous studies have illustrated that humans can learn - ostensibly

462 simultaneously - from multiple sources of information and tend to organise this information in a

463 hierarchical fashion such that the source which is currently of highest value has the greatest

464 influence on a learner's behaviour (Daw et al., 2006). Here we extend this work by showing that the

465 primary source, at the top of the hierarchy, is more heavily influenced by modulation of the

466 dopamine system, thus suggesting a graded involvement of the dopamine system according to a

467 source's status in the "learning hierarchy". Extant studies (Daw et al., 2006) suggest that such

468 learning hierarchies are flexible and can be rapidly remodelled according to a source's current value.

469 The success of our orthogonalization of social versus individual and primary versus secondary

470 learning depended on a within-subjects design, wherein the status (primary or secondary) of the

471 learning source varied only between participants. Although our study was therefore not optimised

472 for studying the rapid remodelling of learning hierarchies, our results pave the way for future studies

473 to investigate whether the impact of dopaminergic modulation of learning from a particular source

474 quickly changes according to the source's current status in the learning hierarchy.

476 In sum, in previous paradigms that dissociate social and individual learning, the social information

477 comprised a secondary or additional information source, differing from individual information both

478 in terms of its social nature (social/individual) and status (secondary/primary). We here provide

479 evidence that dissociable effects of dopaminergic manipulation on different learning types are

480 better explained by primary versus secondary status, than by social versus individual nature.

481 Specifically, we showed that, relative to placebo, haloperidol reduced learning rates relating to

482 learning from the primary, but not secondary, source of information irrespective of social versus 
bioRxiv preprint doi: https://doi.org/10.1101/2021.12.01.470554; this version posted December 3, 2021. The copyright holder for this

preprint (which was not certified by peer review) is the author/funder, who has granted bioRxiv a license to display the preprint in perpetuity. It is made available under aCC-BY 4.0 International license.

483 individual nature. Results illustrate that social and individual learning share a common dependence

484 on dopaminergic mechanisms when they are the primary learning source. 
485

Table I

486

Participant information

\begin{tabular}{|c|c|c|c|c|c|}
\hline & $\begin{array}{c}\text { Individual-primary } \\
\text { group } \\
(\mathrm{n}=15) \\
\text { Mean (SD) }\end{array}$ & $\begin{array}{l}\begin{array}{l}\text { Social-primary } \\
\text { group }\end{array} \\
(n=16) \\
\text { Mean (SD) }\end{array}$ & $t(1,29)$ & $X^{2}(1, N=31)$ & $p$ \\
\hline $\begin{array}{l}\text { Gender } \\
\text { ( } n \text { males: } n \\
\text { females) }\end{array}$ & $7: 8$ & $8: 8$ & & 0.034 & 0.853 \\
\hline Age & $25.600(5.448)$ & $25.625(4.745)$ & 0.014 & & 0.989 \\
\hline VWM & $80.333(6.016)$ & $76.354(7.823)$ & 1.580 & & 0.125 \\
\hline BMI & $24.016(2.807)$ & $22.625(2.606)$ & 1.431 & & 0.114 \\
\hline
\end{tabular}

487 Note: SD refers to standard deviation, VWM refers to verbal working memory span, BMI refers to

488 body mass index. Age, gender, BMI and VWM did not significantly differ between the groups. 
Materials and Methods

490

491

Subjects

492

Subjects ( $n=43$, aged 19 to 42 years, mean (SD) = $26(6.3) ; 19$ female) were recruited from the University of Birmingham and surrounding areas in Birmingham city, via posters, email lists and social media. Four participants dropped out of the study after completing the first day. A further five participants could not complete the second test day, due to university-wide closures and a restriction of data collection. In total, 43 participants completed one session, with 33 participants completing both test days. However, Bayes exclusion factors were reported for interactions of interest, to avoid the possibility of type 2 error. The study was in line with the local ethical guidelines approved by the local ethics committee (ERN_18_1588) and in accordance with the Helsinki Declaration of 1975.

\section{General procedure}

503 The study protocol was pre-registered (see Open Science Framework (OSF) https://osf.io/drmjb for study design and a priori sample size calculations). All participants attended a preliminary health screening session with a qualified clinician, followed by two test sessions with an interval of one to a maximum of four weeks between testing session. The health screening session, lasting approximately one hour, started with informed consent, followed by a medical screening. Participants were excluded from further participation if they met any of the exclusion criteria. Participants then completed a battery of validated questionnaire measures (see Appendix 1 for inclusion/exclusion criteria, questionnaire measures, medical symptoms, and mood ratings). Both

511 test days (1-4 weeks post health screening) followed the same procedure, starting with informed

512 consent, followed by a medical screening. Participants were then administered capsules (by a

513 member of staff not involved in data collection) containing either $2.5 \mathrm{mg}$ haloperidol (HAL) or

514 placebo (PLA), in a double-blind, placebo-controlled, cross-over design. Participants were told to

515 abstain from alcohol and recreational drugs in the 24 hours prior to testing and from eating in the

516 two hours prior to capsule intake.

5181.5 hours after capsule intake, participants commenced a battery of behavioural tasks, including a 519 probabilistic learning paradigm (Go-NoGo learning (Frank \& O'Reilly, 2006)) and a measure of verbal 520 working memory (Sternberg, 1969). The social learning task was started approximately 3 hours post521 capsule administration, within the peak of HAL blood plasma concentration. HAL dosage and 522 administration times were in line with similar studies which demonstrated both behavioural and 
523 psychological effects of haloperidol (Bestmann et al., 2014; Frank \& O’Reilly, 2006). Both test days

524 lasted approximately 5.5 hours in total, with participants starting at the same time of day for both

525 sessions. Blood pressure, mood and medical symptoms were monitored throughout each day:

526 before capsule intake, three times during the task battery and after finishing the task battery. On

527 completion of the second session, participants reported on which day they thought they had taken

528 the active drug or placebo. Participants received monetary compensation on completion of both

529 testing sessions, at a rate of $£ 10$ per hour, with the opportunity to add an additional $£ 5$ based on

530 their performance during the learning task.

\section{Behavioural task}

533 Participants completed a modified version of a social learning task (Cook et al., 2014), first

534 developed by Behrens and colleagues (Behrens et al., 2008). The task was programmed using

535 MATLAB R2017b (The MathWorks, Natick, MA). Participants were randomly allocated to one of two

536 groups. For both groups, participants completed 120 trials on both test days. The task lasted

537 approximately 35 minutes, including instructions. Before the main task, participants completed a

538 step-by-step on-screen practice task (10 trials) in which they learnt to choose between the two

539 options to obtain a reward and learned that the "advice" represented by the frame(s) could help in

540 making the correct choice in some phases. In our previous work with the individual-primary

541 condition alone, we demonstrated that social dominance significantly predicts social, but not

542 individual, learning (Cook et al., 2014). Thus, showing that participants maintain a conceptual

543 distinction between the social and individual learning sources. In the current study we investigated

544 whether participants, maintained this conceptual distinction by requiring participants to complete a

545 short quiz (3 questions), testing their knowledge, after the practice task. Participants were required

546 to repeat the practice round until they achieved $100 \%$ correct score in the quiz, meaning that all

547 participants understood the structure of the task, and that the red shape represented social

548 information. Furthermore, after the experiment, participants completed a feedback questionnaire

549 (Appendix 5). Answers confirmed that participants understood the difference between, and paid

550 attention to both, individual and social sources of information. Participants were informed as to

551 whether they had earned a $f 5$ bonus after the second session. Due to ethical considerations, all

552 participants received the bonus.

\section{Individual-primary group}

555 On each trial participants were required to choose between a blue or green box to gain points.

556 Participants could also use an additional, secondary, source of information - a red frame surrounding 
557 either the blue or green box - to help make their decision. Participants were informed (see Appendix

5585 for instruction scripts) that the frame represented the most popular choice made by a group of

559 participants who had previously completed the task. They were also informed that the task followed

560 'phases' wherein sometimes the blue, but at other times the green choice, was more likely to result

561 in reward and sometimes the social information predominantly indicated the correct box, but at

562 other times it predominantly surrounded the incorrect box (Fig.1A). After making their choice

563 participants received outcome information in the form of a blue or green indicator. The indicator

564 primarily informed participants about whether the blue or green box had been rewarded on the

565 current trial. Whether the social information surrounded the correct or incorrect box could,

566 secondarily, be inferred from the indicator. For example, if the red frame indicated that the social

567 group had chosen the blue shape, and the blue shape was shown to be correct, participants could

568 infer that the social information had therefore been correct on that trial. Both the probability of

569 reward associated with the blue/green stimuli and the utility of the social information, varied

570 according to separate probabilistic schedules, with participants randomly assigned to one of four

571 groups (Appendix 2). For both individual and social information, the probabilistic schedules featured

572 stable phases, where the probability of reward was constant, and volatile phases, in which the

573 probability switched every 10-20 trials. This feature of the task design was included to capture

574 potential effects of dopaminergic modulation on adaptation to environmental volatility (Cook et al.,

575 2019). Participants were informed that correct choices would be rewarded, and thus to aim to

576 accumulate points to obtain a reward at the end of the experiment. Although probabilistic schedules

577 for Day 2 were the same as Day 1, there was variation in the trial-by-trial outcomes and advice. In

578 addition, to prevent participants from transferring learned stimulus-reward associations from Day 1

579 to Day 2, different coloured stimuli were employed on the second session: participants viewed

580 blue/green squares with advice represented as a red frame on Day 1 and yellow/purple squares with

581 advice represented as a blue frame on Day 2.

582

583 Social-primary group

584 For the social-primary group the social information source was the primary source of learning. On

585 each trial participants were presented with two grey placeholders. One placeholder was filled with a

586 red box, indicating the group's choice. Blue/green frames then appeared around the placeholders.

587 As in the individual-primary group, participants were informed that the task followed 'phases'

588 wherein sometimes going with, but at other times going against, the group's choice was more likely

589 to result in reward and sometimes the blue frame predominantly indicated the correct box, whereas

590 at other times the green frame predominantly indicated the correct box. After making their choice 
591 participants received outcome information in the form of a tick/cross indicator. The indicator

592 primarily informed participants about whether the social group had been rewarded (and thus going

593 with them would have resulted in points scoring but going against them would not) on the current

594 trial. Whether the blue(green) frame surrounded the correct or incorrect option could, secondarily,

595 be inferred from the indicator. As in the individual-primary task, both the probability of reward

596 associated with the blue/green stimuli and the utility of the social information varied according to

597 probabilistic schedules (Appendix 2). All other aspects of the task structure were the same as

598 previously described in the individual-primary task group.

599

\section{Data analysis}

601 All analyses were conducted using MATLAB R2017b (The MathWorks, Natick, MA) and Bayesian

602 analyses using JASP (JASP Team (2020). JASP (Version 0.14) [Computer software]). Linear mixed

603 models were fitted to data using RStudio (RStudio Team (2020). RStudio: Integrated Development

604 for R. RStudio, PBC, Boston, MA). In the instance of data not meeting assumptions of normality (as

605 assessed by Kolmogorov-Smirnov testing), data were square-root-transformed. Learning rate $\alpha$

606 values were square-root transformed. We used the standard $p<.05$ criteria for determining if

607 significant effects were observed, with a Holm correction applied for unplanned multiple

608 comparisons, to control for type I family-wise errors. In addition, effect sizes and beta weights for

609 linear mixed model analysis are reported.

610

611 Data pre-processing

612 Datasets were excluded based on the following: accuracy $<50 \%$ under placebo, chose the same side

613 (left/right) or colour on $>80 \%$ trials, incomplete datasets (less than 120 trials completed). Two

614 subjects were excluded, resulted in a final sample of $n=31$, with behavioural data for both testing

615 days, and $n=41$, with data for one day only (see Appendix 4i for analysis).

616

617 Computational modelling framework

618 Participant responses were modelled using an adapted Rescorla-Wagner learning model (Rescorla \&

619 Wagner, 1972). The model relies on the assumption that updates to choice behaviour are based on

620 prediction errors, i.e., the difference between an expected and the actual outcome. Participants

621 were assumed to update their beliefs about outcomes based on sensory feedback (perceptual

622 model), and to use this feedback to make decisions about the next action (response model). Model

623 fitting was performed using scripts adapted from the TAPAS toolbox (Diaconescu et al., 2014)

624 (scripts available at OSF link https://tinyurl.com/b3c7d2zb). A systematic comparison of eight 
separate models (Appendix 3 for full details regarding model fitting and model comparison) showed that the exceedance probability of this particular model was $\sim 1$. This demonstrates (relative) evidence in favour of the conclusion that, the current model, with separate learning rates for primary and secondary information, and volatile and stable phases, provided the best fit to participant choice data and that the data likely originated from the same model for both HAL and PLA treatment conditions (Supplemental Fig 2). Further model validation, including simulation of data and parameter recovery, provided further support for the choice of computational model (Appendix 3).

\section{Perceptual model}

635 The Rescorla-Wagner predictors used in our learning models consisted of a modified version of a simple learning model, with one free parameter, the learning rate $\alpha$, varying between 0 and 1 .

$$
V_{(i+1)}=V_{i}+\alpha\left(r_{i}-V_{i}\right)
$$

640 According to this model the predicted value $\left(V_{i}\right)$ is updated on each trial based on the prediction 641 error (PE), or the difference between the actual and the expected reward $\left(r_{i}-V_{i}\right)$, weighted by the 642 learning rate $\alpha . \alpha$ thus captures the extent to which the PE updates the estimated value on the next 643 trial. In line with previous work (Cook et al., 2019), we used an extended version of this learning 644 model, with separate $\alpha$ values for volatile and stable environmental phases. In a stable environment, 645 learning rate will optimally be low, and reward outcomes over many trials will be taken into account. 646 In a volatile environment, however, an increased learning rate is optimal, as more recent trials are 647 used to update choice behaviour (Behrens et al., 2007). Furthermore, we simultaneously ran two 648 Rescorla-Wagner predictors in order to estimate parameters relating to learning from primary and 649 secondary information sources. Consequently, our model generated the predicted value of going 650 with the primary source (going with the blue frame for the individual-primary group, going with the 651 group for the social-primary group; $V_{-}$primary(i+1)) and the predicted value of the secondary 652 information (going with the group recommendation for the individual-primary group, going with the 653 blue frame for the social-primary group; $V_{-}$secondary (i+1)) and provided four $\alpha$ estimates: $\alpha_{\text {primary_stable, }}$

657 Our response model assumed that participants integrated learning from both primary and secondary sources. The action selector predicts the probability that the primary information (blue choice/ 
group choice) will be rewarded on a given trial and was based on the softmax function (TAPAS toolbox), adapted by Diaconescu and colleagues (Diaconescu et al., 2014). This response model is adapted from that used by Cook and colleagues (Cook et al., 2019) and reproduced here with permission. The value of primary and secondary information was combined using the following:

$$
V_{\_} \text {primary }(i+1)=\zeta\left(V_{-} \text {secondary_advice_weighted }(\mathrm{i}+1)\right)+(1-\zeta)\left(V_{\_} \text {primary }(\mathrm{i}+1)\right)
$$

wherein $\zeta$ is a parameter that varies between individuals, and which controls the weighting of secondary relative to primary sources of information. $V_{\text {_secondary_advice_weighted(i+1) }}$ comprises the advice provided by the secondary information (the red and blue frames, for individual-primary and socialprimary groups respectively) weighted by the probability of advice accuracy $\left(V_{-}\right.$secondary $\left.(i+1)\right)$ in the context of making a choice to go with the primary information (the blue and red box for the individual-primary and social-primary groups respectively). That is:

$$
V_{-} \text {secondary_advice_weighted }(i+1)=\mid \operatorname{advice}-V_{-} \text {secondary }(i+1) \mid
$$

where advice from the red frame equals 0 for blue and 1 for green, and advice from the blue frame equals 0 for going with the red box and 1 for going against the red box. For example, for a participant in the social-primary group, if the blue frame advised them to go with the red box (the group choice $)$ and the probability of advice accuracy was estimated at $80 \%\left(V_{-}\right.$secondary $\left.(i+1)=0.80\right)$, the probability that the choice to go with the group will be rewarded, inferred from secondary learning, would be $0.8\left(V_{\text {_secondary_advice_weighted }(i+1)}=|0-0.8|=0.8\right)$. The probability that this integrated belief would determine participant choice was described by a unit square sigmoid function, describing how learned belief values are translated into choices.

683

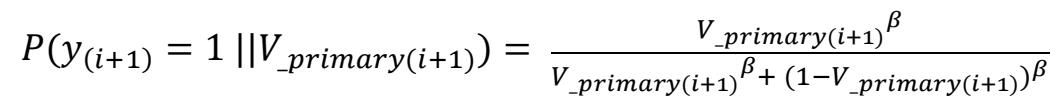

Here, responses are coded as $y_{(i+1)}=1$ when selecting the primary option (going with the blue and red box for the individual-primary and social-primary groups respectively), and $y_{(i+1)}=0$ when selecting the alternative (going with the green box and going against the red box for the individual-primary and social-primary groups respectively). The participant-specific free parameter $\beta$, the inverse of the decision temperature, describes the extent to which estimated value of choices determines actual participant choice: as $\beta$ decreases, decision noise increases and decisions become more stochastic; as $\beta$ increases, decisions become more deterministic towards the higher value option. 


\section{Significance tests for estimated model parameters}

694 Parameters were fitted separately for each participant's choice data. Learning rate $(\alpha)$ was

695 estimated for each participant, for primary and secondary learning, for volatile and stable phases, on

696 both test days, resulting in 8 estimated learning rates per participant. $\beta$ values were also estimated

697 for each participant on both treatment days, resulting in two $\beta$ values per participant. Effects-coded

698 mixed model linear analyses were carried out, to allow for inclusion of subject as a random factor

699 thus ensuring that between-participant variation in $\alpha$ could be controlled for. Fixed factors were

700 drug (HAL, PLA), information type (primary, secondary), volatility (volatile, stable) and group

701 (individual primary, social-primary), with the inclusion of random intercepts for participant: group

$702 x$ information $\mathrm{x}$ drug $\mathrm{x}$ volatility $+1 \mid$ subject.

703

704 Repeated-measures analysis of variance (RM-ANOVA) for linear mixed effects models was carried out using the Satterthwaite approximation for degrees of freedom, and the model was fit using maximum likelihood estimation, with a model including random intercepts, but not random slopes, providing the best fit to the data. All analyses were repeated with and without the inclusion of age, $\mathrm{BMI}$ and baseline working memory as covariates, with the pattern of results unchanged. Where appropriate, data were transformed to meet assumptions of normality for parametric testing.

\section{Bayesian statistical testing}

Bayesian statistical testing was implemented as a supplement to null hypothesis significance tests, to investigate if null results represent a true lack of a difference between the groups (Dienes, 2014), using JASP software, based on the R package "BayesFactor" (Rouder et al., 2012). The JASP

715 framework for repeated measures ANOVA was used (Van Den Bergh et al., 2020), whereby exclusion

716 Bayes factors were obtained for predictors of interest. The exclusion Bayes factor $\left(\mathrm{BF}_{\text {excl }}\right)$ for a given

717 predictor or interaction quantifies the change in odds from the prior probability that the predictor is

718 included in the regression model, to the probability of exclusion in the model after seeing the data

$719\left(\mathrm{BF}_{\text {excl }}\right)$. Bayes factors were computed by comparing all models with a predictor against all models

720 without that predictor, i.e., comparing models that contain the effect of interest to equivalent

721 models stripped of the effect. For example, an exclusion Bayes factor for an effect of 3 for a given

722 predictor $\mathrm{i}$ can be interpreted as stating that, models which exclude the predictor $\mathrm{i}$, are 3 times more

723 likely to describe the observed data than models which include the predictor. In short, the exclusion

724 Bayes factor is interpreted as the evidence given the observed data for excluding a certain predictor

725 in the model and can be used as evidence to support null results. For all Bayesian analyses, the Bayes

726 factor quantifies the relative evidence for one theory or model over another. We followed the 
classification scheme used in JASP (Lee \& Wagenmakers, 2013) to classify the strength of evidence given by the Bayes factors, with $\mathrm{BF}_{\text {excl }}$ between one and three considered as weak evidence, between three and ten as moderate evidence and greater than ten as strong evidence for the alternative hypothesis respectively.

\section{Acknowledgements}

We would like to acknowledge Ms Lydia Hickman for assistance with data collection and Dr Kasim

736 Qureshi and Dr Hannah Liu for medical screening. Ms Rybicki's role in this project is supported by a

737 Midlands Integrative Biosciences Training Partnership (MIBTP) - Biotechnology and Biological

738 Sciences Research Council (BBSRC) PhD studentship. Dr Cook, Dr Sowden and Ms Schuster were

739 supported by the European Union's Horizon 2020 Research and Innovation Programme under

740 European Research Council (ERC)-2017-STG Grant Agreement No 757583 (Brain2Bee).

\section{Authors' contributions}

743 A.R made substantial contributions to the design of the study, collected and reviewed the papers,

744 conducted the experiment, wrote the manuscript, and approved the final draft. S.S and B.S 745 contributed to data collection. J.C contributed to the conception and design of the study, wrote the 746 manuscript, provided a critical review of the manuscript, and approved the final draft. All authors 747 edited the final draft.

\section{Competing interests}




\section{References}

Apps, M. A. J., Rushworth, M. F. S., \& Chang, S. W. C. (2016). The anterior cingulate gyrus and social cognition: tracking the motivation of others. Neuron, 90(4), 692-707. https://doi.org/10.1016/j.neuron.2016.04.018

Balsters, J. H., Apps, M. A. J., Bolis, D., Lehner, R., Gallagher, L., \& Wenderoth, N. (2017). Disrupted prediction errors index social deficits in autism spectrum disorder. Brain, 140(1), 235-246. https://doi.org/10.1093/brain/aww287

Behrens, T. E. J., Hunt, L. T., \& Rushworth, M. F. S. (2009). The computation of social behavior. Science, 324(5931), 1160-1164. https://doi.org/10.1126/science.1169694

Behrens, T. E. J., Hunt, L. T., Woolrich, M. W., \& Rushworth, M. F. S. (2008). Associative learning of social value. Nature, 456(7219), 245-249. https://doi.org/10.1038/nature07538

Behrens, T. E. J., Woolrich, M. W., Walton, M. E., \& Rushworth, M. F. S. (2007). Learning the value of information in an uncertain world. Nature Neuroscience, 10(9), 1214-1221. https://doi.org/10.1038/nn1954

Benoit-Marand, M., Borrelli, E., \& Gonon, F. (2001). Inhibition of dopamine release via presynaptic D2 receptors: Time course and functional characteristics in vivo. Journal of Neuroscience, 21(23), 9134-9141. https://doi.org/10.1523/jneurosci.21-23-09134.2001

Bestmann, S., Ruge, D., Rothwell, J., \& Galea, J. M. (2014). The role of dopamine in motor flexibility. Journal of Cognitive Neuroscience, 27(2), 365-376. https://doi.org/10.1162/jocn_a_00706

Biele, G., Rieskamp, J., \& Gonzalez, R. (2009). Computational models for the combination of advice and individual learning. Cognitive Science, 33(2), 206-242. https://doi.org/10.1111/j.15516709.2009.01010.x

Biele, G., Rieskamp, J., Krugel, L. K., \& Heekeren, H. R. (2011). The neural basis of following advice. PLoS Biology, 9(6). https://doi.org/10.1371/journal.pbio.1001089

Braams, B. R., Güroğlu, B., De Water, E., Meuwese, R., Koolschijn, P. C., Peper, J. S., \& Crone, E. A. (2014). Reward-related neural responses are dependent on the beneficiary. Social Cognitive and Affective Neuroscience, 9(7), 1030-1037. https://doi.org/10.1093/scan/nst077

Brazil, I. A., Hunt, L. T., Bulten, B. H., Kessels, R. P. C., de Bruijn, E. R. A., \& Mars, R. B. (2013). Psychopathy-related traits and the use of reward and social information: A computational approach. Frontiers in Psychology, 4(DEC), 1-11. https://doi.org/10.3389/fpsyg.2013.00952

Bromberg-Martin, E. S., Matsumoto, M., Hong, S., \& Hikosaka, O. (2010). A pallidus-habenuladopamine pathway signals inferred stimulus values. Journal of Neurophysiology, 104(2), 10681076. https://doi.org/10.1152/jn.00158.2010 
Burke, C. J., Tobler, P. N., Baddeley, M., \& Schultz, W. (2010). Neural mechanisms of observational learning. Proceedings of the National Academy of Sciences of the United States of America, 107(32), 14431-14436. https://doi.org/10.1073/pnas.1003111107

Campbell-Meiklejohn, D. K., Bach, D. R., Roepstorff, A., Dolan, R. J., \& Frith, C. D. (2010). How the opinion of others affects our valuation of objects. Current Biology, 20(13), 1165-1170. https://doi.org/10.1016/j.cub.2010.04.055

Campbell-Meiklejohn, D. K., Simonsen, A., Frith, C. D., \& Daw, N. D. (2017). Independent neural computation of value from other people's confidence. Journal of Neuroscience, 37(3), 673-684. https://doi.org/10.1523/JNEUROSCI.4490-15.2016

Camps, M., Cortés, R., Gueye, B., Probst, A., \& Palacios, J. M. (1989). Dopamine receptors in human brain: Autoradiographic distribution of D2 sites. Neuroscience, 28(2), 275-290. https://doi.org/10.1016/0306-4522(89)90179-6

Cook, J. L. (2014). Task-relevance dependent gradients in medial prefrontal and temporoparietal cortices suggest solutions to paradoxes concerning self/other control. Neuroscience and Biobehavioral Reviews, 42, 298-302. https://doi.org/10.1016/j.neubiorev.2014.02.007

Cook, J. L., Den Ouden, H. E. M., Heyes, C. M., \& Cools, R. (2014). The social dominance paradox. Current Biology, 24(23), 2812-2816. https://doi.org/10.1016/j.cub.2014.10.014

Cook, J. L., Swart, J. C., Froböse, M. I., Diaconescu, A. O., Geurts, D. E. M., Den Ouden, H. E. M., \& Cools, R. (2019). Catecholaminergic modulation of meta-learning. ELife, 8, 1-38. https://doi.org/10.7554/eLife.51439

Cooper, J. C., Dunne, S., Furey, T., \& O’Doherty, J. P. (2012). Human dorsal striatum encodes prediction errors during observational learning of instrumental actions. Journal of Cognitive Neuroscience, 24(1), 106-118. https://doi.org/10.1162/jocn_a_00114

Crişan, L. G., Pană, S., Vulturar, R., Heilman, R. M., Szekely, R., Drugă, B., Dragoş, N., \& Miu, A. C. (2009). Genetic contributions of the serotonin transporter to social learning of fear and economic decision making. Social Cognitive and Affective Neuroscience, 4(4), 399-408. https://doi.org/10.1093/scan/nsp019

Daw, N. D., Niv, Y., \& Dayan, P. (2005). Uncertainty-based competition between prefrontal and dorsolateral striatal systems for behavioral control. Nature Neuroscience, 8(12), 1704-1711. https://doi.org/10.1038/nn1560

Daw, N. D., O’Doherty, J. P., Dayan, P., Seymour, B., \& Dolan, R. J. (2006). Cortical substrates for exploratory decisions in humans. Nature, 441(7095), 876-879. https://doi.org/10.1038/nature04766

Delgado, M. R., Frank, R. H., \& Phelps, E. A. (2005). Perceptions of moral character modulate the 
neural systems of reward during the trust game. Nature Neuroscience, 8(11), 1611-1618. https://doi.org/10.1038/nn1575

Diaconescu, A. O., Mathys, C., Weber, L. A. E., Daunizeau, J., Kasper, L., Lomakina, E. I., Fehr, E., \& Stephan, K. E. (2014). Inferring on the intentions of others by hierarchical Bayesian learning. PLoS Computational Biology, 10(9), e1003810. https://doi.org/10.1371/journal.pcbi.1003810

Diaconescu, A. O., Mathys, C., Weber, L. A. E., Kasper, L., Mauer, J., \& Stephan, K. E. (2017). Hierarchical prediction errors in midbrain and septum during social learning. Social Cognitive and Affective Neuroscience, 12(4), 618-634. https://doi.org/10.1093/scan/nsw171

Diederen, K. M. J., Ziauddeen, H., Vestergaard, M. D., Spencer, T., Schultz, W., \& Fletcher, P. C. (2017). Dopamine modulates adaptive prediction error coding in the human midbrain and striatum. Journal of Neuroscience, 37(7), 1708-1720. https://doi.org/10.1523/JNEUROSCI.1979-16.2016

Dienes, Z. (2014). Using Bayes to get the most out of non-significant results. Frontiers in Psychology, 5(July), 1-17. https://doi.org/10.3389/fpsyg.2014.00781

Dolan, R. J., \& Dayan, P. (2013). Goals and habits in the brain. Neuron, 80(2), 312-325. https://doi.org/10.1016/j.neuron.2013.09.007

Ereira, S., Hauser, T. U., Moran, R., Story, G. W., Dolan, R. J., \& Kurth-Nelson, Z. (2020). Social training reconfigures prediction errors to shape Self-Other boundaries. Nature Communications, 11(1), 1-14. https://doi.org/10.1038/s41467-020-16856-8

Ford, C. P. (2014). The role of D2-autoreceptors in regulating dopamine neuron activity and transmission. Neuroscience, 282, 13-22. https://doi.org/10.1016/j.neuroscience.2014.01.025.The

Frank, M. J., \& O'Reilly, R. C. (2006). A mechanistic account of striatal dopamine function in human cognition: psychopharmacological studies with cabergoline and haloperidol. Behav Neurosci, 120(3), 497-517. https://doi.org/10.1037/0735-7044.120.3.497

Frey, A. L., \& McCabe, C. (2020). Effects of serotonin and dopamine depletion on neural prediction computations during social learning. Neuropsychopharmacology, 45(9), 1431-1437. https://doi.org/10.1038/s41386-020-0678-z

Garvert, M. M., Moutoussis, M., Kurth-Nelson, Z., Behrens, T. E. J., \& Dolan, R. J. (2015). LearningInduced plasticity in medial prefrontal cortex predicts preference malleability. Neuron, 85(2), 418-428. https://doi.org/10.1016/j.neuron.2014.12.033

Gläscher, J., Daw, N., Dayan, P., \& Doherty, J. P. O. (2011). States versus Rewards: Dissociable neural prediction error signals underlying model-based and model-free reinforcement learning. Neuron, 66(4), 585-595. https://doi.org/10.1016/j.neuron.2010.04.016.States 
Glimcher, P. W., \& Bayer, H. M. (2005). Midbrain dopamine neurons encode a quantitative reward prediction error signal. Neuron, 103(11), 2304-2312. https://doi.org/10.1038/mp.2011.182.doi

Grace, A. A. (2002). Dopamine. In Neuropsychopharmacology: The Fifth Generation of Progress (pp. 120-132).

Haarsma, J., Fletcher, P., Ziauddeen, H., Spencer, T., \& Diederen, K. (2018). Precision weighting of cortical unsigned prediction errors is mediated by dopamine and benefits. BioRxiv, 1-24. https://doi.org/10.1101/288936

Heyes, C. M. (2012). What's social about social learning? Journal of Comparative Psychology, 126(2), 193-202. https://doi.org/10.1037/a0025180

Heyes, C. M., \& Pearce, J. M. (2015). Not-so-social learning strategies. Proceedings of the Royal Society B: Biological Sciences, 282(1802). https://doi.org/10.1098/rspb.2014.1709

Hill, M. R., Boorman, E. D., \& Fried, I. (2016). Observational learning computations in neurons of the human anterior cingulate cortex. Nature Communications, 7, 12722. https://doi.org/10.1038/ncomms12722

Jones, J. L., Esber, G. R., McDannald, M. A., Gruber, A. J., Hernandez, A., Mirenzi, A., \& Schoenbaum, G. (2012). Orbitofrontal cortex supports behavior and learning using inferred but not cached values. Science, 338(6109), 953-956. https://doi.org/10.1126/science.1227489

Kendal, R. L., Boogert, N. J., Rendell, L., Laland, K. N., Webster, M., \& Jones, P. L. (2018). Social learning strategies: Bridge-building between fields. Trends in Cognitive Sciences, 22(7), 651665. https://doi.org/10.1016/j.tics.2018.04.003

Klucharev, V., Hytönen, K., Rijpkema, M., Smidts, A., \& Fernández, G. (2009). Reinforcement learning signal predicts social conformity. Neuron, 61(1), 140-151.

https://doi.org/10.1016/j.neuron.2008.11.027

Laland, K. N. (2004). Social learning strategies. Learning \& Behaviour, 32(1), 4-14. https://doi.org/10.1063/1.470327

Langdon, A. J., Sharpe, M. J., Schoenbaum, G., \& Niv, Y. (2018). Model-based predictions for dopamine. Current Opinion in Neurobiology, 49, 1-7. https://doi.org/10.1016/j.conb.2017.10.006

Lee, M. D., \& Wagenmakers, E. J. (2013). Bayesian cognitive modeling: A practical course. In Bayesian Cognitive Modeling: A Practical Course. Cambridge University Press. https://doi.org/10.1017/CBO9781139087759

Li, J., Delgado, M. R., \& Phelps, E. A. (2011). How instructed knowledge modulates the neural systems of reward learning. Proceedings of the National Academy of Sciences of the United States of America, 108(1), 55-60. https://doi.org/10.1073/pnas.1014938108 
Lidow, M. S., Goldman-Rakic, P. S., Gallager, D. W., \& Rakic, P. (1991). Distribution of dopaminergic receptors in the primate cerebral cortex: Quantitative autoradiographic analysis using $[3 \mathrm{H}]$ raclopride, $[3 \mathrm{H}]$ spiperone and $[3 \mathrm{H}] \mathrm{SCH} 23390$. Neuroscience, 4O(3), 657-671. https://doi.org/10.1016/0306-4522(91)90003-7

Lindström, B., Haaker, J., \& Olsson, A. (2018). A common neural network differentially mediates direct and social fear learning. Neurolmage, 167(March 2017), 121-129. https://doi.org/10.1016/j.neuroimage.2017.11.039

Manning, C., Kilner, J., Neil, L., Karaminis, T., \& Pellicano, E. (2017). Children on the autism spectrum update their behaviour in response to a volatile environment. Developmental Science, 20(5). https://doi.org/10.1111/desc.12435

Menon, M., Jensen, J., Vitcu, I., Graff-Guerrero, A., Crawley, A., Smith, M. A., \& Kapur, S. (2007). Temporal Difference Modeling of the Blood-Oxygen Level Dependent Response During Aversive Conditioning in Humans: Effects of Dopaminergic Modulation. Biological Psychiatry, 62(7), 765-772. https://doi.org/10.1016/j.biopsych.2006.10.020

Moran, R., Dayan, P., \& Dolan, R. J. (2021). Human subjects exploit a cognitive map for credit assignment. Proceedings of the National Academy of Sciences of the United States of America, 118(4), 1-12. https://doi.org/10.1073/pnas.2016884118

Morgan, T. J. H., Rendell, L. E., Ehn, M., Hoppitt, W., \& Laland, K. N. (2012). The evolutionary basis of human social learning. Proceedings of the Royal Society B: Biological Sciences, 279(1729), 653662. https://doi.org/10.1098/rspb.2011.1172

Nicolle, A., Klein-Flügge, M. C., Hunt, L. T., Vlaev, I., Dolan, R. J., \& Behrens, T. E. J. (2012). An agent independent axis for executed and modeled choice in medial prefrontal cortex. Neuron, 75(6), 1114-1121. https://doi.org/10.1016/j.neuron.2012.07.023

O’Doherty, J. P., Dayan, P., Friston, K., Critchley, H., \& Dolan, R. J. (2003). Temporal difference models and reward-related learning in the human brain. Neuron, 38(2), 329-337. https://doi.org/10.1016/S0896-6273(03)00169-7

Pessiglione, M., Seymour, B., Flandin, G., Dolan, R. J., \& Frith, C. D. (2006). Dopamine-dependent prediction errors underpin reward-seeking behaviour in humans. Nature, 442(7106), 10421045. https://doi.org/10.1038/nature05051

Rendell, L., Fogarty, L., Hoppitt, W. J. E., Morgan, T. J. H., Webster, M. M., \& Laland, K. N. (2011). Cognitive culture: Theoretical and empirical insights into social learning strategies. Trends in Cognitive Sciences, 15(2), 68-76. https://doi.org/10.1016/j.tics.2010.12.002

Rescorla, R. A., \& Wagner, A. R. (1972). A theory of Pavlovian conditioning: Variations in the effectiveness of reinforcement and nonreinforcement. In Clasical conditioning II: current 
research and theory (pp. 64-99). Appleton Century Crofts.

Richerson, P. J., \& Boyd, R. (2005). Not By Genes Alone: How Culture Transformed Human Evolution. The University of Chicago Press.

Roberts, C., Sahakian, B. J., \& Robbins, T. W. (2020). Psychological mechanisms and functions of 5-HT and SSRIs in potential therapeutic change: Lessons from the serotonergic modulation of action selection, learning, affect, and social cognition. Neuroscience and Biobehavioral Reviews, 119(April), 138-167. https://doi.org/10.1016/j.neubiorev.2020.09.001

Rouder, J. N., Morey, R. D., Speckman, P. L., \& Province, J. M. (2012). Default Bayes factors for ANOVA designs. Journal of Mathematical Psychology, 56(5), 356-374. https://doi.org/10.1016/j.jmp.2012.08.001

Sadacca, B. F., Jones, J. L., \& Schoenbaum, G. (2016). Midbrain dopamine neurons compute inferred and cached value prediction errors in a common framework. ELife, 5(MARCH2016), 1-13. https://doi.org/10.7554/eLife.13665

Schmitz, Y., Benoit-Marand, M., Gonon, F., \& Sulzer, D. (2003). Presynaptic regulation of dopaminergic neurotransmission. Journal of Neurochemistry, 87(2), 273-289. https://doi.org/10.1046/j.1471-4159.2003.02050.x

Schultz, W. (2007). Behavioral dopamine signals. Trends in Neurosciences, 30(5), 203-210. https://doi.org/10.1016/j.tins.2007.03.007

Schultz, W., Dayan, P., \& Montague, P. R. (1997). A neural substrate of prediction and reward. Science, 275(5306), 1593-1599. https://doi.org/10.1126/science.275.5306.1593

Sharpe, M. J., \& Schoenbaum, G. (2018). Evaluation of the hypothesis that phasic dopamine constitutes a cached-value signal. Neurobiology of Learning and Memory, 153(July 2017), 131136. https://doi.org/10.1016/j.nlm.2017.12.002

Sternberg, S. (1969). Memory-scanning: mental processes revealed by reaction-time experiments. American Scientist, 57(4), 421-457.

Sutton, R., \& Barto, A. G. (2018). Reinforcement learning: An introduction. (2nd ed.). MIT press. Tarantola, T., Kumaran, D., Dayan, P., \& De Martino, B. (2017). Prior preferences beneficially influence social and non-social learning. Nature Communications, 8(1), 817. https://doi.org/10.1038/s41467-017-00826-8

Templeton, J. J., Kamil, A. C., \& Balda, R. P. (1999). Sociality and social learning in two species of corvids: The pinyon jay (Gymnorhinus cyanocephalus) and the Clark's nutcracker (Nucifraga columbiana). Journal of Comparative Psychology, 113(4). https://doi.org/10.1037/07357036.113.4.450

Van Den Bergh, D., Van Doorn, J., Marsman, M., Draws, T., Van Kesteren, E. J., Derks, K., Dablander, 
bioRxiv preprint doi: https://doi.org/10.1101/2021.12.01.470554; this version posted December 3, 2021. The copyright holder for this

preprint (which was not certified by peer review) is the author/funder, who has granted bioRxiv a license to display the preprint in perpetuity. It is made available under aCC-BY 4.0 International license.

F., Gronau, Q. F., Kucharský, Š., Gupta, A. R. K. N., Sarafoglou, A., Voelkel, J. G., Stefan, A., Ly, A., Hinne, M., Matzke, D., \& Wagenmakers, E. J. (2020). A tutorial on conducting and interpreting a bayesian ANOVA in JASP. Annee Psychologique, 120(1), 73-96.

https://doi.org/10.3917/anpsy1.201.0073

Zhang, L., \& Gläscher, J. (2020). A brain network supporting social influences in human decisionmaking. Science Advances, 6(34), 1-20. https://doi.org/10.1126/sciadv.abb4159 


\section{Appendix 1}

\section{Inclusion criteria}

Participant is willing and able to give informed consent for participation in the study. Aged between 18 and 45.

BMI in the range of $18.5-29.5$

Resting blood pressure in the range of 90/60 (low) to 140/90 (high)

Electrocardiogram QT (heart rate corrected) interval $<.42$

\section{Exclusion criteria}

Participation in another drug study in the 3 weeks previous.

Personal or first-degree family history of cardiovascular disease, specifically hypotension, arrhythmias or valvular disease, stroke

Neurological abnormalities or traumas, kidney disease or liver disease Inherited blood conditions

Psychiatric or psychological conditions (including depression and anxiety disorders)

Known learning disability

Anybody found to have an elongated Q-T interval following single lead ECG examination Low heart rate

Low or high blood pressure

Any regular medication - excluding the oral contraceptive pill

Recent recreational drugs use or alcohol and drug dependency

Known allergy to any medication

Current pregnancy or breastfeeding

Previous participant in a drug study

Lack of sleep in last 24 hours.

Lack of food or drink in last 12 hours

Primary sensory impairment (e.g., uncorrected visual or hearing impairment)

Lactose intolerant

Insufficient English to be able to consent to take part in the study

\section{Baseline cognitive measures and mood ratings}

Approximately one week prior to drug/placebo administration, participants completed a battery of self-report questionnaire measures: Autism Spectrum quotient $(A Q)^{1}$, Toronto Alexithymia Scale $(\text { TAS 20) })^{2}$, Behavioural Inhibition/Activation Scale (BIS-BAS) ${ }^{3}$, the Depression Anxiety and Stress Scale (DASS 21) ${ }^{4}$, Interpersonal Reactivity Index (IRI) ${ }^{5}$, Beck's Depression Inventory (BDI) ${ }^{6}$ and Body Perception Questionnaire (BPQ) ${ }^{7}$. Self-report questionnaire scores are summarised in Supplemental Table 1. The individual-primary group did not differ significantly on any measure from the social- 
primary group. The group that received HAL on day 1 did not differ significantly on any of the baseline measures from the group that received PLA on day $1(p<0.05)$. Mood and fatigue were monitored three times per day during each test day, i) before capsule intake, ii) two hours postcapsule intake upon start task battery, and iii) upon completion of the task battery. The mood ratings consisted of the Positive and Negative Affect Scale (PANAS) ${ }^{8}$. A self-report scale was used to monitor fatigue. $24 \%$ of participants reported that they did not know on which day they had taken an active drug. Out of the remaining participants, $84 \%$ of participants correctly reported that they thought they had received an active drug. No adverse side effects were reported. Blood pressure, heart rate and blood oxygenation levels were monitored five times over the course of the testing days; before drug/placebo administration, and then at one, two and three and a half hour intervals thereafter. Measures were taken for a final time immediately before the end of the testing day.

Supplemental Table 1. Self-report questionnaire scores for the individual-primary and social-primary groups $(n=33)$

\begin{tabular}{r|cccc}
$\begin{array}{r}\text { Self-report } \\
\text { questionnaires }\end{array}$ & $\begin{array}{c}\text { Individual- } \\
\text { primary group }\end{array}$ & $\begin{array}{l}\text { Social-primary } \\
\text { group }\end{array}$ & $t(31)$ & $p$-value \\
\hline AQ & $9.412(4.556)$ & $6.500(4.179)$ & 1.910 & 0.065 \\
TAS-20 & $39.529(6.947)$ & $40.313(7.981)$ & -0.301 & 0.765 \\
BIS-BAS & $50.647(6.855)$ & $51.125(5.536)$ & -0.219 & 0.828 \\
DASS-StresS & $3.176(4.231)$ & $3.875(2.306)$ & -0.583 & 0.723 \\
DASS-Depression & $1.353(2.178)$ & $1.938(2.516)$ & -0.715 & 0.564 \\
IRI & $1.706(1.863)$ & $2.313(3.005)$ & -0.702 & 0.480 \\
BDI & $66.235(15.114)$ & $66.375(10.645)$ & -0.031 & 0.976 \\
$B P Q$ & $3.176(3.746)$ & $3.438(2.732)$ & -0.227 & 0.822 \\
& $52.176(29.473)$ & $46.688(18.650)$ & 0.635 & 0.221
\end{tabular}

Note: Mean (standard deviation) scores are reported. Significance level for the between-group differences are reported. Autism Spectrum quotient (AQ) ${ }^{1}$, Toronto Alexithymia Scale (TAS 20) ${ }^{2}$, Behavioural Inhibition/Activation Scale (BIS-BAS) ${ }^{3}$, the Depression Anxiety and Stress Scale (DASS $21)^{4}$, Interpersonal Reactivity Index (IRI) ${ }^{5}$, Beck's Depression Inventory (BDI) ${ }^{6}$ and Body Perception 62 Questionnaire (BPQ) 7 .

63

Positive and negative affect (PANAS) scores were submitted to separate RM-ANOVAs, with withinsubjects (WS) factors time (baseline/start testing/end testing) and drug (HAL/PLA). For both positive and negative scores, a main effect of time was observed. Both positive $(F(2,62)=8.286, p<0.001$, $\left.\eta_{p}{ }^{2}=0.211\right)$, and negative scores decreased over time $\left(F(2,62)=6.020, p=0.004, \eta_{p}{ }^{2}=0.163\right)$. A drug by time interaction was observed for positive scores $\left(F(2,62)=7.353, p=0.001, \eta_{p}{ }^{2}=0.192\right)$, with 
simple effects analysis demonstrating that positive scores decreased over time under haloperidol ( $p$ $<0.001)$, but not placebo $(p=0.994)$. A main effect of drug was observed on negative scores $(F$ $\left.(1,31)=4.749, \mathrm{p}=0.037, \eta_{\mathrm{p}}^{2}=0.133\right)$, with higher negative affect scores under haloperidol $\left(\bar{x}\left(\sigma_{\bar{x}}\right)=\right.$ $10.771(0.557)$ compared with placebo $\left(\bar{x}\left(\sigma_{\bar{x}}\right)=9.491(0.557)\right)$. fatigue) were submitted to a RM-ANOVA, with WS factors time (T1-T5) and drug (HAL/PLA). A main effect of time was observed, with fatigue rising across time $\left(F(4,88)=6.652, p<0.001, \eta_{p}{ }^{2}=0.232\right)$. No main or interaction effect(s) involving drug were observed.

\section{Appendix 2}

\section{Randomisation groups}

82

For both the social-primary and individual-primary group, the probability of reward associated with the blue/green stimuli (individual information) and the red stimuli (social information) were governed by different pseudo-randomisation schedules, adapted from Behrens et al ${ }^{9}$. Schedules were counterbalanced between participants to ensure that learning could not be explained in terms of differences in learning between schedules with increased/decreased, or early/late occurring, volatility. The individual-primary group (schedules 1,3 ) were sub-divided into two groups, such that half started with predominantly correct social information, and half with predominantly incorrect social information, with the same true for the social-primary group (schedules 2,4 ). The primary information source was always less volatile overall compared to the secondary information source, irrespective of whether it was social or individual. To give an example, the randomisation schedule for group 1 was the same as that employed by Behrens et al ${ }^{9}$. During the first 60 trials, the individual reward history was stable, with a 75\% probability of blue being correct. During the next 60 trials, the reward history was volatile, switching between $80 \%$ green correct and $80 \%$ blue correct every 20 trials. Meanwhile, during the first 30 trials, social information was stable, with $75 \%$ of choices being correct. During the next 40 trials, the social information was volatile, switching between $80 \%$ incorrect and $80 \%$ correct every 10 trials. During the final 50 trials, social information was once again stable, with $85 \%$ of choices being incorrect. Randomisation schedules for groups 2,3 , and 4 were inverted and counterbalanced versions of schedule 1 (Suppl. Fig. 1). 

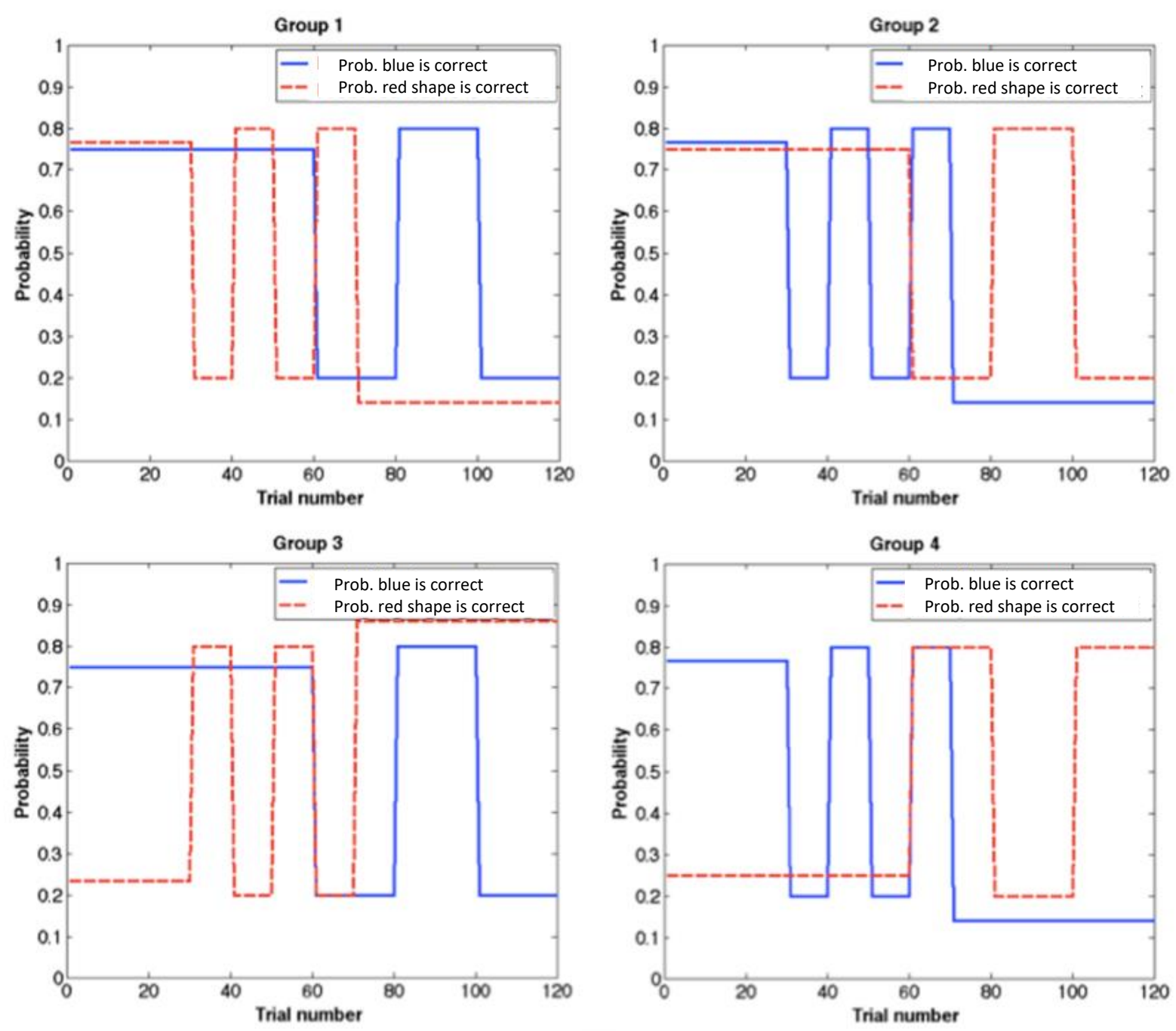

Suppl. Figure 1. Randomisation schedules. The probability of reward varied according to probabilistic schedules, including stable and volatile blocks for both the probability of blue being correct and the probability of the social information indicating the correct answer. Probability schedules were counterbalanced between participants. Solid blue lines show the probability of blue being the correct choice, dashed red lines show the probability of the social information being correct. Schedules 1-4 are displayed here. 
111 Optimisation of free parameter values was performed as per Cook and colleagues ${ }^{10}$, using a quasi-

112 Newton optimisation algorithm specified in TAPAS toolbox - quasinewton_optim_config.m. The

113 function maximised the log-joint posterior density over all parameters given the data and the

114 generative model. $\alpha$ values were estimated in logit space (see tapas_logit.m), i.e., a logistic sigmoid

115 transformation of native space (tapas_logit $(x)=\ln (x /(1-x)) ; x=1 /(1+\exp (-\operatorname{tap}$ as_logit $(x))))$. An

116 uninformative prior, allowing for individual differences in learning rate was used for $\alpha$ : tapas_logit

$117(0.2,1)$, with a variance of 1 . Initial values were set at logit $(0.5,1)$, with a variance of 1 . Initial values

118 were allowed to vary, to allow for inter-individual differences in prior preferences for the extent to

119 which individual would conform to the group choice. The prior for $\beta$ was set to log (48), with a

120 variance of 1 , and the prior for $\zeta$ was set at 0 with a variance of $10^{2}$ (logit space), i.e., an equal

121 weighting for information derived from primary and secondary learning (0.5). Prior choices were

122 based on previous work ${ }^{10}$. Maximum-a-posteriori (MAP) estimates for all model parameters were

123 calculated using the HGF toolbox version 3 (https://osf.io/398w4/files/). All code used is adapted

124 from the open-source software package TAPAS (available

125 at http://www.translationalneuromodeling.org/tapas).

Model comparison

We based our choice of perceptual model on previous work by Cook and others ${ }^{10}$, wherein a

130 systematic comparison of three alternative models was conducted, to determine which best explained observed choice behaviour. Here we repeated Cook et al.'s model comparison and added four further extensions of the classic model, thus we compared eight alternative models in total. A formal model comparison was carried out using Bayesian model selection using the VBA toolbox ${ }^{11}$.

Data were initially analysed with eight models. All models were variations of the classic RescorlaWagner model. Group level Bayesian model selection (BMS) was used to evaluate which model provided the (relative) best fit to the observed data. The VBA toolbox ${ }^{12}$, specifically random-effects BMS (using the VBA_groupBMC_btwConds.m function), was utilised. Random effects group BMS computes an approximation of the model evidence relative to the other models, i.e., the probability

140 of the data $y$ given a model $m, \mathrm{p}(y / m)$, with log model evidence here approximated with $\mathrm{F}$ values.

141 The posterior probability that a model has generated the observed data, relative to other models is 
142 estimated, and the exceedance probability, or the likelihood that a given model is more likely than

143 other included models in the set, is estimated. Analysis across both conditions allows us to test the

144 hypothesis that the same model produced observed data under both haloperidol and placebo conditions.

Model 1 was a classic Rescorla-Wagner model: (PE). value and secondary value learning sources:

Model 3 had a single learning rate $\alpha$ for primary/secondary learning, but separate learning rates for volatile and stable blocks:

161

$$
\begin{gathered}
V_{\text {primary }(i+1)}=V_{-p \operatorname{primary}(i)}+\alpha_{\text {primary }} \varepsilon_{i} \\
V_{\text {secondary }(i+1)}=V_{\text {secondary }(i)}+\alpha_{\text {secondary }} \varepsilon_{i}
\end{gathered}
$$

162

163 Model 4 had four separate learning rates $\alpha$ for volatile and stable and primary and secondary

164 learning:

$$
\begin{gathered}
V_{\text {primary }(i+1)}=V_{\text {primary }(i)}+\alpha_{\text {primary_volatile }} \varepsilon_{i}+\alpha_{\text {primary_stable }} \varepsilon_{i} \\
V_{\text {secondary }(i+1)}=V_{\text {secondary }(i)}+\alpha_{\text {secondary_volatile }} \varepsilon_{i}+\alpha_{\text {secondary_stable }} \varepsilon_{i}
\end{gathered}
$$

165

166 As an exploratory measure, we further extended Models 1-4 to include separate learning rates

167 corresponding to learning from rewarded trials and unrewarded trials separately, i.e., learning from 168 wins and losses. 
Model 5:

$$
V_{(i+1)}=V_{i}+\alpha_{-} \text {reward } \varepsilon_{i}+\alpha_{-} \text {unreward } \varepsilon_{i}
$$

172

Model 6:

$$
\begin{aligned}
& V_{\text {primary }(i+1)}=V_{\text {primary }(i)}+\alpha_{\text {primary_reward }} \varepsilon_{i}+\alpha_{\text {primary_unreward }} \varepsilon_{i} \\
& V_{\text {secondary }(i+1)}=V_{\text {secondary }(i)}+\alpha_{\text {secondary_reward }} \varepsilon_{i}+\alpha_{\text {secondary_unreward }} \varepsilon_{i}
\end{aligned}
$$

Model 7:

$$
\begin{gathered}
V_{(i+1)}=V_{i}+\alpha_{\text {_volatile_reward }} \varepsilon_{i}+\alpha_{\text {sstable_reward }} \varepsilon_{i}++\alpha_{\_} \text {volatile_unreward } \varepsilon_{i} \\
+\alpha_{\text {sstable_unreward }} \varepsilon_{i}
\end{gathered}
$$

176

Model 8:

181

$$
\begin{aligned}
V_{\text {secondary }(i+1)} & =V_{\text {Secondary }(i)} V_{(i+1)}+\alpha_{\text {secondary_volatile_reward }} \varepsilon_{i}+\alpha_{\text {secondary_stable_reward }} \varepsilon_{i} \\
& +\alpha_{\text {secondary_volatile_unreward }} \varepsilon_{i}+\alpha_{\text {secondary_stable_unreward }} \varepsilon_{i}
\end{aligned}
$$

184 We ran a between-groups model comparison, to ensure that the same model could explain the observed data under both placebo and haloperidol. When comparing all models, Model 4 performed best, with an exceedance probability approaching 1 . The exceedance probability that the same model (Model 4) had produced data under both conditions was equal to 1. For condition 1 (placebo),

188 the posterior probabilities that the observed data had produced the model was equal to 10.329 for

189 Model 3 and 12.998 for Model 4, with the probability that the data was produced by the winning 190 model $p(\mathrm{H} 1 \mid \mathrm{y})=0.762$. For group 2 (haloperidol), Model 4 had a posterior probability of 15.417

$191(\mathrm{p}(\mathrm{H} 1 \mid \mathrm{y})=0.998)$. For the between-groups assessment, the posterior probability $\mathrm{p}(\mathrm{H} 1 \mid \mathrm{y})=0.999$

192 and the protected exceedance probability $(\varphi)$ was equal to 0.999 . 
198
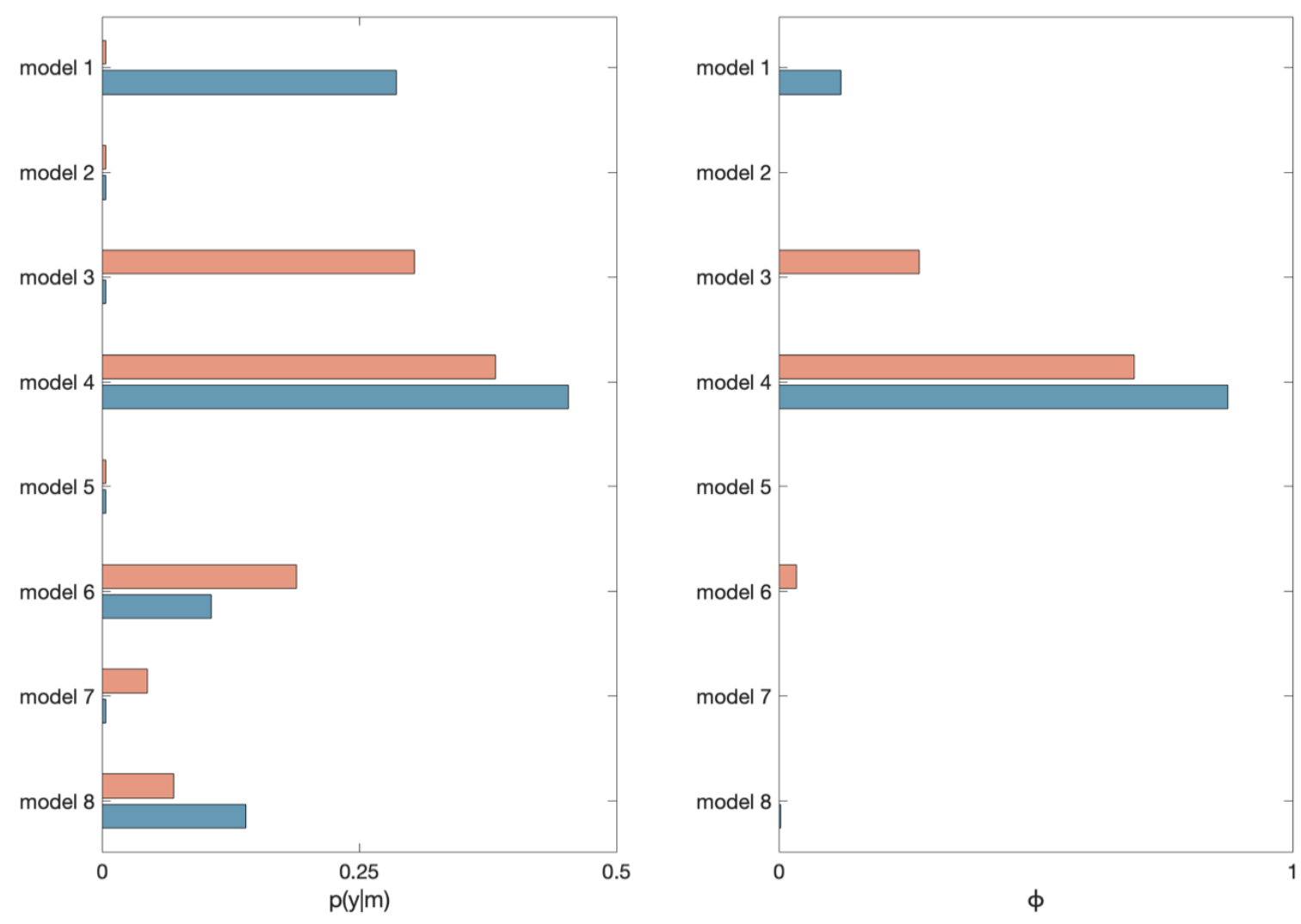

Suppl. Figure 2. Model comparison. Results from random-effects Bayesian model selection. Exceedance Probability and posterior model probability for models 1-8. $\mathrm{p}(\mathrm{y} \mid \mathrm{m})=$ posterior model probability, $\varphi=$ exceedance probability, $\mathrm{HAL}=$ blue, $\mathrm{PLA}=$ red .

204 To demonstrate that the chosen model (model 4) accurately described participant behaviour, we

205 simulated response data for each participant, using estimated model parameter values

206 (tapas_simModel.m). Accuracy did not significantly differ between actual and simulated accuracy for

207 PLA ( $t=-0.866, p=0.394)$ or HAL conditions $(t=-0.280, p=0.781)$ (Suppl. Fig. $3 A)$. Simulated and

208 calculated accuracy were significantly correlated for each participant, under both placebo $(r=0.487$, $p=0.005)$ and haloperidol conditions $(r=0.712, p<.001)$ (Suppl. Fig. 3B). 
bioRxiv preprint doi: https://doi.org/10.1101/2021.12.01.470554; this version posted December 3,2021 . The copyright holder for this

preprint (which was not certified by peer review) is the author/funder, who has granted bioRxiv a license to display the preprint in perpetuity. It is made available under aCC-BY 4.0 International license.

\section{Supplemental Figure 3}

213 A
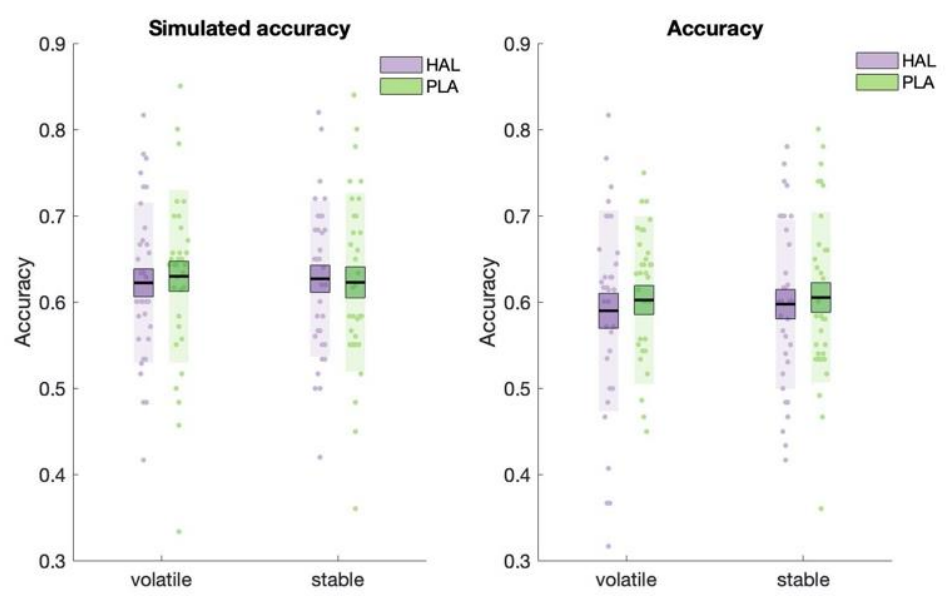

B
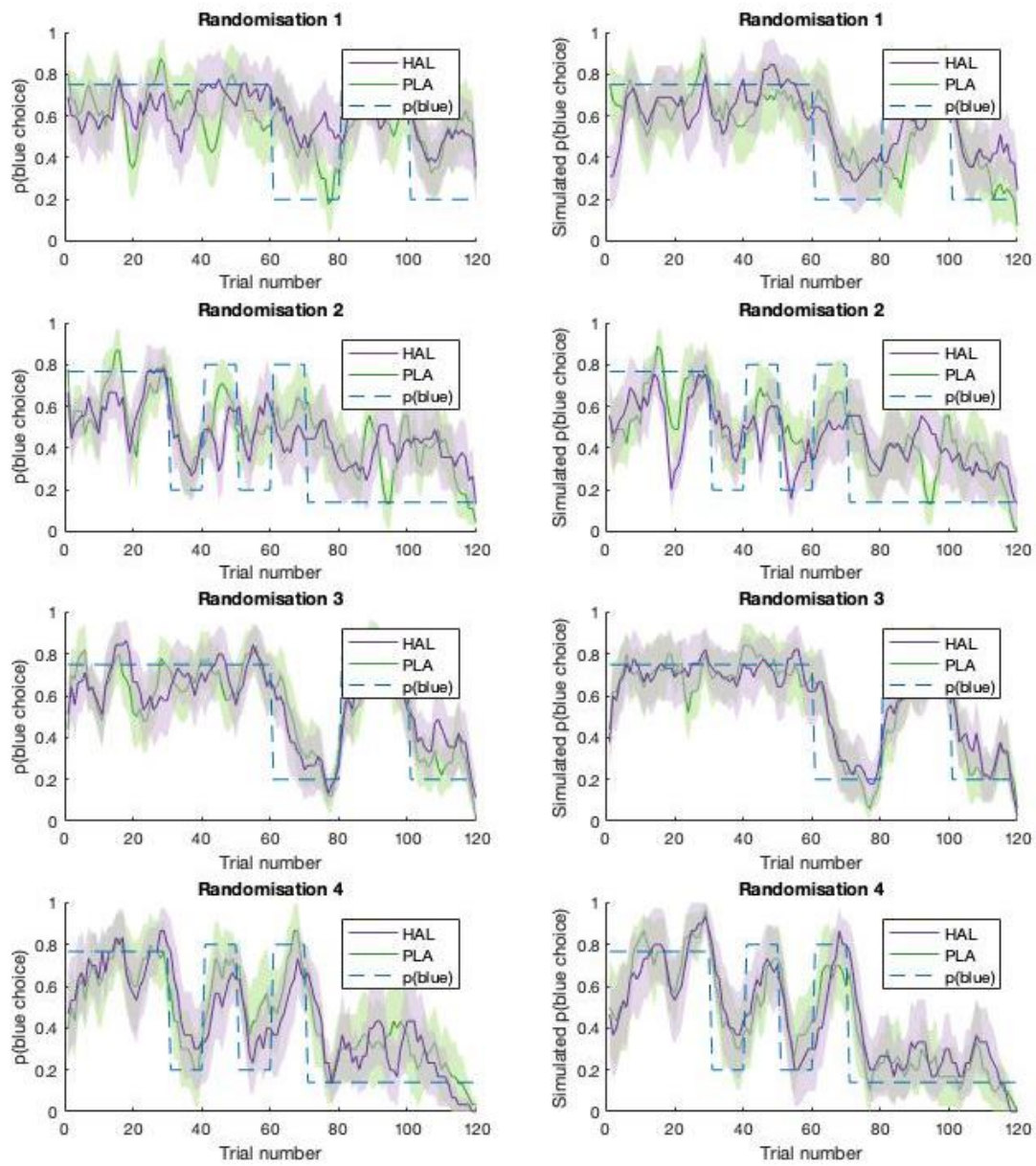

Suppl. Fig. 3. A. Model simulations (left) and participant response data (right). Mean accuracy is displayed separately for volatile and stable environmental phases, under HAL (purple) and PLA (green). Boxes = standard error of the mean, shaded region = standard deviation, individual datapoints are displayed. $\mathrm{HAL}=$ haloperidol, PLA $=$ placebo.

B. Participant data (left) juxtaposed against model simulations (right) Running average, across 5 trials of blue choices for probabilistic randomisation schedules 1 to 4 . Shaded region = standard error of the mean. 
To ensure that parameter estimates could be recovered, model parameters were estimated from simulated data for each participant, separately for HAL and PLA conditions. All recovered parameters correlated significantly with estimated parameters under both treatment conditions (all $p<0.001$ ).

\section{Appendix 4}

\section{Extended statistical analyses}

i. Learning rate analysis $(n=41)$

228 A RM-ANOVA, with (square-root transformed) learning rate $(\alpha)$ as the DV and predictors information source, volatility, drug and group was carried out on estimates from the mixed model analysis which included all participants who completed at least one study day $(N=41)$. A significant main effect of information was observed $\left(\mathrm{F}(1,234)=3.944, \mathrm{p}=0.048\right.$, beta estimate $\left(\sigma_{\bar{x}}\right)=0.019(0.010), \mathrm{t}=1.986$, $\mathrm{CI}[0-0.04]$ ), with higher mean values for $\alpha_{\text {primary }}$ (estimate $(S E)=0.429(0.018)$ ) compared with $\alpha_{\text {secondary }}$ (estimate $\left.(\mathrm{SE})=0.391(0.018)\right)$.

A significant volatility by information interaction $(F(1,234)=4.676, p=0.032$, beta estimate $(S E)=$ $0.021(0.010), t=-2.162, \mathrm{Cl}[0-0.04])$ was observed. Post hoc comparisons revealed that, under stable phases, $\alpha_{\text {primary }}$ values (estimate $(S E)=0.461(0.023)$ ) were significantly greater than $\alpha_{\text {secondary }}$ (estimate $\left.(S E)=0.381(0.023), z=2.933, p_{\text {holm }}=0.007\right)$, with no difference between $\alpha$ in volatile environmental phases $\left(z=-0.125, p_{\text {holm }}=0.901\right)$. No main effect of group was observed, however, there was a significant information by group interaction $(F(1,234)=32.471, p<0.001$, beta estimate $(\mathrm{SE})=0.05(0.010), \mathrm{t}=5.700, \mathrm{Cl}[0.04-0.07])$. Post hoc comparisons revealed that, for the individual-primary group, $\alpha_{\text {primary }}$ (estimate $(S E)=0.455(0.026)$ ) was significantly greater than

244 however, $\alpha_{\text {secondary }}$ (estimate (SE) $=0.475(0.025)$ ) was significantly greater than $\alpha_{\text {primary }}$ (estimate (SE)

$\left.245=0.404(0.025), z=2.667, p_{\text {holm }}=0.015\right)$.

247 A significant volatility by group interaction was observed $(F(1,234)=4.168, p=0.042$, beta estimate $248(\mathrm{SE})=0.020(0.010), \mathrm{t}=2.042, \mathrm{Cl}[0-0.04])$. For the individual-primary group, $\alpha_{\text {volatile }}$ (estimate $(\mathrm{SE})=$ $2490.351(0.026)$ ) showed a non-significant trend towards being lower than $\alpha_{\text {stable }}$ (estimate (SE) $=0.411$ $\left.250(0.026), z=-2.192, p_{\text {holm }}<0.057\right)$. For the social-primary group, however, $\alpha_{\text {volatile }}$ (estimate $(S E)=$ $2510.449(0.025))$ and $\alpha_{\text {stable }}$ (estimate $\left.(S E)=0.431(0.025)\right)$ did not significantly differ $\left(z=0.672, p_{\text {holm }}=\right.$ 252 0.502). 
254 Most importantly, as with the analysis reported in the main text, a significant drug by information 255 interaction was observed $(F(1,234)=3.727, p=0.054$, beta estimate $(S E)=0.01(0.1), t=1.69, \mathrm{Cl}$

$256[0.00-0.04]$. Post hoc comparisons demonstrated that, under PLA there was a significant difference between $\alpha_{\text {primary }}$ (estimate $(S E)=0.451(0.023)$ and $\alpha_{\text {secondary }}$ (estimate $(S E)=0.375(0.023), z=2.727$, $p_{\text {holm }}=0.026$, uncorrected $p=0.006$ ). This difference was nullified under HAL $\left(\alpha_{\text {primary }}\right.$ estimate $(S E)=$ $0.408(0.023)$ and $\alpha_{\text {secondary }}$ (estimate $\left.(S E)=0.407(0.023)\right)\left(z=0.040, p_{\text {holm }}=0.968\right.$, uncorrected $p=$ $0.968)$.

There was no significant group $x$ information source $x$ drug interaction $(F(1,234)=0.029, p=0.866$, beta estimate $(\mathrm{SE})=-0.002(0.010), \mathrm{t}=-0.169, \mathrm{Cl}[-0.02-0.02])$.

266 An analysis of accuracy was conducted in participants who had completed both study days ( $n=31$ ), to explore whether there was any systematic variation as a function of randomization schedule, and across drug and placebo conditions and volatile and stable phases. A RM-ANOVA, with withinsubjects factors drug (HAL, PLA) and volatility (stable, volatile), and between-subjects factor group (social-primary, individual-primary) and randomisation schedule (1-4), demonstrated no difference in accuracy between haloperidol $\left(\bar{x}\left(\sigma_{\bar{x}}\right)=0.601(0.011)\right)$, and placebo $\left(\bar{x}\left(\sigma_{\bar{x}}\right)=0.614(0.011) ; \mathrm{F}(1,27)\right.$

$\left.272=1.161, p=0.291, \eta_{p}{ }^{2}=0.041\right)$. However, a significant main effect of schedule was observed $(\mathrm{F}$ $\left.273(3,27)=3.004, p=0.048, \eta_{p}{ }^{2}=0.250\right)$, with the lowest accuracy observed for schedule $1\left(\bar{x}\left(\sigma_{\bar{x}}\right)=\right.$

$2740.558(0.019)$. Although accuracy for schedule 1 was lower than for schedule $2\left(\bar{x}\left(\sigma_{\bar{x}}\right)=0.619\right.$ $\left.275(0.018), \mathrm{t}(27)=-2.358, \mathrm{p}_{\text {holm }}=0.129\right)$, schedule $3\left(\bar{x}\left(\sigma_{\bar{x}}\right)=0.614(0.018), \mathrm{t}(27)=(-2.162), \mathrm{p}_{\text {holm }}=\right.$ $2760.159)$ and schedule $4\left(\bar{x}\left(\sigma_{\bar{x}}\right)=0.637(0.020), \mathrm{t}(27)=-2.748, \mathrm{p}_{\mathrm{holm}}=0.063\right)$; these differences were 277 no longer significant after correction for multiple comparisons. Mean accuracy for schedules 2-4 did 278 not significantly differ from each other (all p-values $=1.000$ ). In addition, there was a significant 279 interaction effect between schedule and volatility $\left(F(3,27)=7.527, p<0.001, n p^{2}=0.455\right)$. For all 280 schedules except for schedule 3 , there was no significant difference in accuracy between volatile and stable phases (all p>0.05). However, for schedule 3, accuracy was significantly higher for volatile $\left(\bar{x}\left(\sigma_{\bar{x}}\right)=0.675(0.022)\right.$ over stable phases $\left(\bar{x}\left(\sigma_{\bar{x}}\right)=0.533(0.022), \mathrm{t}(27)=(3.656), \mathrm{p}_{\mathrm{holm}}=0.027\right)$.

283 Accuracy was significantly higher for the social-primary group $\left(\bar{x}\left(\sigma_{\bar{x}}\right)=0.629\right.$ (0.013), compared with 284 the individual-primary group $\left(\bar{x}\left(\sigma_{\bar{x}}\right)=0.586(0.013), \mathrm{F}(1,29)=5.196, \mathrm{p}=0.030, \mathrm{n}_{\mathrm{p}}{ }^{2}=0.152\right)$ and no other main effects or interactions were observed (all $p>0.05$ ). 
A backwards regression with PLA accuracy as the dependent variable, and $\alpha_{\text {primary }}$ and $\alpha_{\text {secondary }}$ (collapsed across volatile and stable phases), initial values $V_{\text {primary(i) }}$ and $V_{\text {secondary }(i)}, \beta$ and $\zeta$ as predictors, was carried out. PLA accuracy was marginally significantly predicted by a model with $\alpha_{\text {secondary }}$ as a single predictor $(R=0.347, F(1,29)=3.981, p=0.055)$. Under haloperidol, a backward regression with HAL accuracy as the dependent variable, and $\alpha_{\text {primary, }} \alpha_{\text {secondary, }}, V_{\text {primary }(i)}$, $V_{\text {secondary }(i)}, \beta$ and $\zeta$ as predictors, revealed that HAL accuracy was significantly predicted by the full model. Within the model, $\alpha_{\text {primary }}$ was the only significant predictor (Suppl. Table 2). Removing predictors did not significantly improve the fit of the model $\left(R^{2}\right.$ change $<0.001, F$ change $(1,25)=-$ $0.064, p=1.000)$.

Supplemental Table 2

Coefficients from regression model with HAL accuracy as the dependent variable.

\begin{tabular}{|c|c|c|c|c|c|}
\hline & $\beta$ & $\beta$ (SEM) & $\begin{array}{c}\text { standardised } \\
\beta\end{array}$ & $\mathbf{t}$ & $p$ \\
\hline constant & 0.431 & 0.089 & & 4.840 & $<0.001$ \\
\hline $\boldsymbol{\alpha}_{\text {primary }}$ & 0.195 & 0.077 & 0.431 & 2.532 & $0.018^{*}$ \\
\hline $\boldsymbol{\alpha}_{\text {secondary }}$ & 0.076 & 0.119 & 0.127 & 0.642 & 0.527 \\
\hline$V_{\text {primary }(i)}$ & 0.121 & 0.090 & 0.230 & 1.342 & 0.192 \\
\hline$V_{\text {secondary }(i)}$ & 0.033 & 0.131 & 0.050 & 0.249 & 0.806 \\
\hline $\boldsymbol{\beta}$ & 0.002 & 0.001 & 0.329 & 1.698 & 0.102 \\
\hline$\zeta$ & 0.045 & 0.043 & 0.189 & 1.066 & 0.297 \\
\hline
\end{tabular}

304 To further investigate the neurochemical mechanisms underlying the observed decrease in $\alpha_{\text {primary }}$ under haloperidol, we measured performance on a probabilistic Go, No-go control task, adapted

306 from Frank and colleagues ${ }^{13}$ and presented using MATLAB R2017b. Participants were presented with 3074 different stimuli, each with a probabilistic value of reward $(80 \%, 60 \%, 40 \%, 20 \%)$ and instructed to 308 accumulate as many points as possible and to avoid losing points, achieved by selecting or

309 withholding a response to the given stimuli. For example, if selected, stimuli A would result in

310 gaining a point on $80 \%$ of trials and losing a point on $20 \%$ of trials. Participants were informed that

311 points would be rewarded with monetary compensation; however, due to ethical considerations, all 
312 participants were awarded $£ 5$ at the end, regardless of task performance. Participants first

313 completed 4 blocks of a practice stage, where single stimuli were presented ( 40 trials/block, with

314 each stimulus presented 10 times per block). Reward feedback was provided, allowing learning of

315 the probabilistic value of each stimulus. This was followed by 6 testing blocks ( 40 trials/block)

316 displaying either single stimuli (training stimuli) or novel pairs of stimuli on each trial, whereby

317 participants were required to respond based on the combined probabilistic value of the pairs.

318 Testing blocks contained positive pairs with a high associated probabilistic reward value, equal pairs

319 (equally probable reward value), and negative pairs, with a high probabilistic value for punishment.

320 Participants could respond via a 'Go' (space bar press) or 'No-Go' (withhold response) response.

321 Feedback was not provided during testing blocks. In all trials, a fixation cross was presented for 250-

$322750 \mathrm{~ms}$, followed by stimuli presentation for $1000 \mathrm{~ms}$ and a response period for $250 \mathrm{~ms}$. Task

323 performance was calculated as the difference in 'Go' response for stimuli (novel pairs and single

324 stimuli) with a high probability of reward under HAL and PLA conditions, for each participant

325 separately.

327 Previous research (using a similar low, acute dose of haloperidol) resulted in enhancement of

328 learning from positive reinforcement, indexed by an increase in learning from positive feedback ${ }^{13}$,

329 suggested to be mediated via pre-synaptic antagonistic effects on phasic dopamine (DA) signalling.

330 As an exploratory measure, participants were stratified into two subgroups based on performance

331 during this task; those with a higher change in 'Go' performance for high reward trials under

332 haloperidol, and those with a lower change in 'Go' performance under haloperidol, relative to

333 placebo. For the participants who demonstrated increased 'Go' performance under haloperidol $(n=$

334 12), a significant drug by information effect was observed on the main behavioural task $(F(1,10)=$

$\left.3354.773, p=0.054, \eta_{p}{ }^{2}=0.323\right)$. However, this effect was not observed in participants with reduced

336 'Go' performance under haloperidol $\left(n=19 ; F(1,17)=2.001, p=0.175, \eta_{p}{ }^{2}=0.105\right)$. Thus, suggesting

337 that the observed effect of haloperidol on learning rate for primary information was driven by a

338 subgroup of participants who exhibited increased 'Go' performance under haloperidol (relative to

339 placebo). Given that such effects on Go performance have been linked to pre-synaptic antagonistic

340 effects on phasic DA signalling ${ }^{13}$ these results suggest that the effects we observed on $\alpha_{\text {primary }}$ are

341 likely mediated by effects of haloperidol on phasic DA signalling.

343 While an increase in Go performance suggests effects of haloperidol on phasic dopamine release,

344 the effects of haloperidol can also result in a reduction in tonic dopamine signalling ${ }^{14}$. These tonic

345 effects are commonly indexed by a slowing of response ${ }^{15,16}$. Indeed, haloperidol had a significant 
effect on (log) reaction time (RT), with higher reaction times observed under haloperidol $\left(\bar{x}\left(\sigma_{\bar{x}}\right)=\right.$ $1.580(0.147)$ seconds $(s))$ when compared with placebo $\left(\bar{x}\left(\sigma_{\bar{x}}\right)=1.242(0.150), \mathrm{p}=0.002, \eta^{2}=\right.$ 0.292). We therefore investigated whether there was a relationship between $\Delta R T$ and $\Delta \alpha$ under haloperidol. A median split $(\Delta R T)$ resulted in two subgroups of participants. Separate RM-ANOVAs, with (square root) learning rate estimates $(\alpha)$ as the dependent variable, and information, volatility and task group as the predictor variables were carried out for each subgroup. For the subgroup of participants who showed the greatest increase in RT (slowing of response) under haloperidol $(n=15)$, the drug by information interaction no longer reached significance $\left(F(1,13)=0.106, p=0.750, \eta_{p}{ }^{2}=\right.$ 0.008). The opposite pattern of results was observed for the subgroup of participants $(n=16)$ with a $\Delta R T$ below the median change (a reduced slowing of response under haloperidol): here a significant drug by information interaction effect was observed $\left(F(1,14)=10.846, p=0.005, \eta_{p}^{2}=0.437\right)$. Results show that, for the subgroup of participants who showed the greatest slowing of response $(\Delta R T)$, haloperidol did not significantly affect learning rates. Given that response slowing has been linked to tonic dopamine this pattern of results further reinforces the idea that our observed effects on $\alpha_{\text {primary }}$ are likely mediated by effects of haloperidol on phasic, not tonic, DA.

364 Randomisation schedule (1-4) and drug day (i.e., haloperidol administered on testing day 1 or 2) were included as predictor variables in all analyses (with both $n=31$ and $n=41$ samples), with no main/interaction effect(s) observed (all $F<1$, all $p>0.05$ ). Additionally, testing session was used to check for the presence of practice effects. Testing session (session 1 or 2 ) was included as a predictor variable in all analysis, with no main/interaction effect(s) observed (all $F<1$, all $p>0.05$ ).

vi. Effects of baseline verbal working memory (VWM) on model parameters

372 As there is evidence to suggest that effects of dopamine manipulation are dependent on baseline DA

373 synthesis, with working memory capacity shown to predict dopamine synthesis in healthy adults ${ }^{17}$,

374 participants completed a visual working memory (VWM) task, adapted from the Sternberg VWM

375 Task (Sternberg, 1969), and programmed using MATLAB R2017b. Participants were first presented

376 with instructions followed by practice trials. Upon completion of the practice trials, participants

377 completed 60 experimental trials across 5 blocks. On each trial, a fixation cross was displayed in the

378 centre of screen (fixation duration varied randomly between 500-1000 ms). Then participants were

379 presented with a list of letters, (varying between 5 - 9 consonants in length, with letters randomly

380 selected from the alphabet on each trial) for $1000 \mathrm{~ms}$, followed by a blue fixation cross for $3000 \mathrm{~ms}$. 
381 Following this, a single test letter was displayed (for a maximum of $4000 \mathrm{~ms}$ ), requiring participants

382 to determine whether the letter was taken from the previously displayed list. For $50 \%$ of trials, the

383 letter had been present on the previous list and on $50 \%$ of trials, it had not. Participants responded

384 by pressing 1-3 on the keyboard (1 - Yes, 2 - No, 3 - Unsure). The total task duration was

385 approximately 10 minutes. Responses (accuracy) and response time (time from test letter displayed

386 until participant response) were recorded for each trial. We then stratified participants into high and

387 low verbal working memory (VWM) groups, based on mean baseline (under placebo) accuracy

388 scores. VWM (high/low) was included as a predictor in a mixed model analysis $(n=31)$. A Type III

389 RM-ANOVA conducted on model estimates revealed a significant interaction between VWM and

390 information type $(F(1,189)=5.932, p=0.016$, beta estimate $(S E)=0.026(0.010), t=2.436, \mathrm{Cl}[0.00-$

$3910.05])$ with planned contrasts revealing that, for low VWM participants, $\alpha_{\text {secondary }}$ values $\left(\bar{x}\left(\sigma_{\bar{x}}\right)=\right.$

3920.364 (0.031) were significantly lower than $\alpha_{\text {primary }}$ values $\left(\bar{x}\left(\sigma_{\bar{x}}\right)=0.447(0.031) ; z(30)=2.820\right.$,

393 pholm $\left._{1}=0.010\right)$. There was no significant difference between $\alpha_{\text {primary }}$ and $\alpha_{\text {secondary }}$ for high VWM

394 participants $\left(z(30)=-0.641, p_{\text {holm }}=0.522\right)$. No other main or interaction effects of VWM on $\alpha$ values

395 were observed (all $F<0.01$, all $p>0.05$ ). Additionally, the pattern of results was unchanged from the

396 previous analysis excluding VWM, with the drug by information interaction effect remaining

397 significant $(F(1,189)=3.967, p=0.048$, beta estimate $(S E)=0.021(0.010), t=1.992, \mathrm{Cl}[0.00-$

398 0.04]). Finally, while including baseline VWM as continuous predictor variable in a RM-ANOVA, no

399 main or interaction effect(s) of VWM on $\alpha$ values were observed. Additionally, neither gender, age

400 nor BMI interacted with any outcome variables (all $F<0.01$, all $p>0.05$ ). Results suggest that the

401 observed decrease in $\alpha_{\text {primary }}$ under haloperidol is not related to variation in working memory

402 capacity.

403

404

405

406

407

408 


\section{Appendix 5}

411

Instruction scripts

\section{i. Individual-primary group}

414

Welcome. You have a choice: either choose the blue shape or the green shape. One shape is correct - guessing which one it is will give you points. To help you to choose, one of the shapes is filled with red. This indicates the most popular choice selected by a group of 4 people who previously played this task. When the question mark appears, try picking a shape by pressing the left or right keyboard buttons. [Participant responds]

420

Feedback: After you make a choice, a tick or cross will appear in the middle. This tells you if the group of previous players were correct or incorrect.

423 Here they think the blue shape (filled with red) will be correct. Try picking a shape now. [Participant responds]

Blue is correct! This means that this time the others got it right.

Things happen in phases in this game. The game could be in a phase where the blue shape is more likely to be correct. Have another go. [Participant responds]

And blue again! It certainly looks as though you are in a blue phase but make sure you pay attention to what the right answers are because the phase that you are in can change at any time. Here's a tip - ignore which side of the screen the shapes are on - it's the colour that is important! [Participant responds]

The others got it right again. It looks like, right now, you could be in a phase where the group's information is useful. Perhaps these are trials from the end of their experiment, when they had developed a pretty good idea of what was going on. Be careful though because we have mixed up the order of the other people's trials so that their choices will also follow phases. Try again. Perhaps the other shape is right this time? [Participant responds]

Green! This time the green shape was right! The chance of each shape being right or wrong will change as you play, so pay attention! The group were incorrect this time. Remember that sometimes you will see less useful information from the group - for example from the beginning of their experiment where they didn't have a very good idea of what was going on. Have another go ... [Participant responds]

This time the green shape was right! The chance of each shape being right or wrong will change as you play, so pay attention. The group were correct too. It looks like, right now, you could be in a phase where the group's information is useful. Try to be as accurate as possible. Getting it right, gives you points. Get enough points and you could earn a silver or even a gold prize! Have another go... [Participant responds]

Things happen in phases in this game. Remember, the tick or cross in the middle tells you if the group were correct or incorrect. That means that the shape with the red box was the correct choice. 
The group were correct this time. The tick in the middle tells you that they picked the correct choice. There will now be a short quiz. Pick one more shape and then we'll head to the real game!

459 [Participant responds]

460

461

\section{ii. Social-primary group}

462

463

Welcome. You have a choice between going with, or against advice from a group. Below you can see a blue and green frame, one frame is filled with a red box: this indicates the most popular choice selected by a group of 4 people who previously played this task. One frame is correct. You can pick the same frame as the group have picked or choose to go against the group's advice. When the question mark appears, make your selection by pressing the left or right keyboard buttons.

468

469 [Participant responds]

Feedback: After you make a choice, a tick or cross will appear in the middle. This tells you if the group of previous players were correct or incorrect.

This time they were correct! This means that the frame filled with the red square was the correct frame.

Here they think the blue frame (filled with red) will be correct. Try picking a frame now. [Participant responds]

The group were correct! This means that this time the others got it right and picked the correct colour.

Things happen in phases in this game. The game could be in a phase where the group are more likely to be correct. Have another go. [Participant responds]

481

482

483

The group were correct again! The blue frame was right again. It certainly looks as though you are in a phase where the group are correct but make sure you pay attention to the feedback because the phase that you are in can change at any time. Blue and green can also go through phases: it looks like you might be in a phase where the blue frame is more likely to be correct. Try again. [Participant responds]

The others got it right again. It looks like, right now, you could be in a phase where the group's information is pretty useful. Perhaps these are trials from the end of their experiment, when they had developed a pretty good idea of what was going on. Be careful though because we have mixed up the order of the other people's trials so that their choices will follow phases. Try again. [Participant responds]

The group were incorrect this time. This time the green frame was correct. The chance of each frame being right or wrong will change as you play, so pay attention! Remember that sometimes you will see less useful information from the group - for example from the beginning of their experiment where they didn't have a very good idea of what was going on. Have another go ... [Participant responds]

The group were correct this time. The chance of each frame being right or wrong will change as you play, so pay attention. Try to be as accurate as possible. Getting it right, gives you points. Get enough points and you could earn a silver or even a gold prize! Have another go... [Participant responds]

Things happen in phases in this game. Remember, the tick or cross in the middle tells you if the group were correct or incorrect. That means that the frame filled with the red was the correct choice. Have another go... [Participant responds] 
509 The group were correct this time. The tick in the middle tells you that they picked the correct choice.

510 There will now be a short quiz. Pick one more time and then we'll head to the real game! [Participant

511 responds]

512

\section{Feedback Questionnaire}

Participants competed a short feedback questionnaire after the behavioural task, consisting of the

516 following questions:

518 1. Did you understand what you were required to do?

519 2. How clear were the task instructions?

520 3. Did you use the group's suggestions (red shape) to help you to make your decision?

521 4. Did you pay attention to which colour (blue/green) was more likely to be correct?

522 5. How difficult did you find the task?

523

$524100 \%$ of participants said that they understood the task instructions and what they were supposed

525 to do. Participants rated on a 5-point Likert scale how often they i) used the group's suggestions (red

526 shape) to help make their decision, comprising the social rating score, and ii) if they paid attention to

527 the colour of the shape (blue/green) that was correct when making their decision (the individual

528 rating score). Social and individual ratings were submitted to separate one-sample t-tests, to ensure

529 that participants in both the individual-primary and social-primary groups were paying attention to

530 both sources of information. Both social $(\mathrm{t}(42)=30.765, \mathrm{p}<0.001)$ and individual ratings $(\mathrm{t}(42)=$

$53129.565, p<0.001)$ were significantly greater than zero.

532 


\section{Supplemental References}

1. Baron-Cohen S, Wheelwright S, Skinner R, Martin J, Clubley E. The Autism-Spectrum Quotient (AQ): Evidence from ... J Autism Dev Disord. 2001;31(1):5-17.

2. Bagby RM, Taylor GJ, Parker JDA. The twenty-item Toronto Alexithymia scale-II. Convergent,

3. Carver CS, White TL. Behavioral Inhibition, Behavioral Activation, and Affective Responses to Impending Reward and Punishment: The BIS/BAS Scales. J Pers Soc Psychol. 1994;67(2):319333. doi:10.1037/0022-3514.67.2.319

4. Lovibond PF, Lovibond SH. Manual for the Depression Anxiety Stress Scales. 2nd ed. (Psychology Foundation, ed.).; 1995.

5. Davis MH. A Mulitdimensional Approach to Individual Differences in Empathy. J Pers Soc Psychol. 1983;44(1):113-126. doi:10.1037/0022-3514.44.1.113

6. Beck AT, Steer RA, Brown G. Beck Depression Inventory-II. In: APA PsycTests; 1996.

7. $\quad$ Porges SW. Body Perception Questionnaire (BPQ) Manual. Stress Int J Biol Stress. 1993;(c):17.

8. Watson D, Clark L, Tellegen A. Development and validation of brief measures of positive and negative affect: the PANAS scales. J Pers Soc Psychol. 1988;54(6):1063-1070. doi:10.1037//0022-3514.54.6.1063.

9. Behrens TEJ, Hunt LT, Woolrich MW, Rushworth MFS. Associative learning of social value. Nature. 2008;456(7219):245-249. doi:10.1038/nature07538

10. Cook JL, Swart JC, Froböse MI, et al. Catecholaminergic modulation of meta-learning. Elife. 2019;8:1-38. doi:10.7554/eLife.51439

11. Stephan KE, Penny WD, Daunizeau J, Moran RJ, Friston KJ. Bayesian model selection for group studies. Neuroimage. 2009;46(4):1004-1017. doi:10.1016/j.neuroimage.2009.03.025

12. Daunizeau J, Adam V, Rigoux L. VBA: A Probabilistic Treatment of Nonlinear Models for Neurobiological and Behavioural Data. PLoS Comput Biol. 2014;10(1). doi:10.1371/journal.pcbi.1003441

13. Frank MJ, O'Reilly RC. A mechanistic account of striatal dopamine function in human cognition: psychopharmacological studies with cabergoline and haloperidol. Behav Neurosci. 2006;120(3):497-517. doi:10.1037/0735-7044.120.3.497

14. Frank MJ, O'Reilly RC. A mechanistic account of striatal dopamine function in human cognition: Psychopharmacological studies with cabergoline and haloperidol. Behav Neurosci. 
15. Grace AA. Dopamine. In: Neuropsychopharmacology: The Fifth Generation of Progress. ; 2002:120-132.

16. Niv Y, Daw ND, Joel D, Dayan P. Tonic dopamine: Opportunity costs and the control of response vigor. Psychopharmacology (Berl). 2007;191(3):507-520. doi:10.1007/s00213-0060502-4

575 17. Cools R, Gibbs SE, Miyakawa A, Jagust W, D'Esposito M. Working memory capacity predicts dopamine synthesis capacity in the human striatum. J Neurosci. 2008;28(5):1208-1212. doi:10.1523/JNEUROSCI.4475-07.2008

578 18. Sternberg S. Memory-scanning: mental processes revealed by reaction-time experiments. Am Sci. 1969;57(4):421-457.

580 